\title{
Isomonodromy, Painlevé transcendents and scattering off of black holes
}

\section{Fábio Novaes and Bruno Carneiro da Cunha}

Departamento de Física, Universidade Federal de Pernambuco, 50670-901, Recife, Pernambuco, Brazil

E-mail: fabiomnsantos@df.ufpe.br, bcunha@df.ufpe.br

ABSTRACT: We apply the method of isomonodromy to study the scattering of a generic Kerr-NUT-(A)dS black hole. For generic values of the charges, the problem is related to the connection problem of the Painlevé VI transcendent. We review a few facts about Painlevé VI, Garnier systems and the Hamiltonian structure of flat connections in the Riemann sphere. We then outline a method for computing the scattering amplitudes based on Hamilton-Jacobi structure of Painlevé, and discuss the implications of the generic result to black hole complementarity.

Keywords: Integrable Equations in Physics, Black Holes, Black Holes in String Theory

ARXIV EPRINT: 1404.5188 


\section{Contents}

1 Introduction 1

2 Killing-Yano and separability $\quad 3$

2.1 Kerr-NUT-(A)dS case 4

2.2 Heun equation from conformally coupled Kerr-NUT-(A)dS 5

3 Scattering, isomonodromy and Painlevé VI 9

4 Flat connections and monodromies $\quad 12$

5 The classical mechanics of monodromies $\quad 18$

$\begin{array}{lll}6 & \text { The generic scattering } & 21\end{array}$

$\begin{array}{lll}7 & \text { Discussion } & 24\end{array}$

A Schlesinger system asymptotics and Painlevé VI 25

\section{Introduction}

The scattering of quantum fields around a classical gravitational background has had an important impact in the study of quantum fields in curved spaces [1]. The classical result for Schwarzschild black holes has helped in the development of the Hawking mechanism, as well as fundamental questions of unitarity of evolution and the exact character of the event horizon as a special place in a black hole background. From the classical perspective, one can also study stability of solutions by these means, treating the metric perturbations as a spin-2 field.

With the advent of the gauge-gravity correspondence, black holes became the prototype of thermal systems, so the problem of scattering had other applications such as the calculation of normal modes and transport properties for the dual theory $[2,3]$. On a more mathematical perspective, the black hole scattering is linked to the monodromy of a Fuchsian equation, [4-6], a fact which drew some attention of late because of its relation to conformal field theory and Liouville field theory [7, 8]. A Fuchsian differential equation is one whose solutions diverge polynomially at singular points. The ocurrence of Fuchsian equations in mathematical physics is pervasive. The mathematical structure behind them is rich enough that one can know enough about their solutions in order to define new functions which have direct physical application.

This article deals with one such structure, the problem of isomonodromic deformations, and its relation to the problem of scattering of black holes. In mathematics, the 
problem was initially studied with the application to the Riemann-Hilbert problem. The latter consists of finding a Fuchsian ordinary differential equation (ODE) with prescribed monodromy data. Soon Poincaré noted that the amount of independent parameters in a Fuchsian ODE with regular singular points was not sufficient to parametrize a generic monodromy group, and then instead one began considering a generic linear system of first order differential equations. Schlesinger [9] found a system of differential equations that enabled one to change the parameters with respect to the positions of the singular points in order to keep the monodromy data fixed. These equations bear his name, and details of the construction can be found in [10].

The construction above has a direct relation to the problem of scattering. Generically, a second order linear differential equation will have two linearly independent solutions. If the differential equation is Fuchsian, then the solutions near a singular point will be asymptotically of the form $\left(z-z_{i}\right)^{\rho_{i}^{ \pm}}$, with the exponents $\rho_{i}^{ \pm}$solutions of the indicial equation near $z_{i}$. These solutions can be continued via Frobenius construction up to another singular point $z_{j}$, where there will be another pair of asymptotic solutions and exponents. As a second order linear ODE has two linearly independent solutions, in general the first set of asymptotic solutions will be a linear combination of the second set. The matrices that relates any such two pair of solutions is called the monodromy data. In a scattering process, if one has, say, a problem of a "purely ingoing" wave in the vicinity of a black hole horizon, then one can use the monodromy data to relate it to a combination of "ingoing" and "outgoing" waves at infinity, and from those coefficients one can compute the scattering amplitudes.

The inverse scattering method has had a very close relationship with integrable structures. This article tries to take advantage of it in two ways: first, one should ask which assumptions one has to impose on the spacetime in order to separate the wave equation and reduce it to a system of Fuchsian equations. Second, one can ask if (and how) the isomonodromic flow helps in obtaining the monodromy data for those Fuchsian equations. As it turns out, the assumptions are exactly that the spacetime has a principal conformal Killing-Yano tensor, which points to a twistorial structure. Solutions of Einstein equations with a cosmological constant with a Killing-Yano tensor are completely determined by their global charges: its mass, angular momentum, and NUT charge. For the second point, we establish that the monodromy data depends on a modified $\tau$-function associated to the isomonodromy flow. Incidentally, the twistorial structure of the isomonodromic flow is more sophisticated and already studied in [11].

The paper is organized as follows. In section two, we perform the reduction of the Klein-Gordon equation into a pair of Fuchsian equations with five regular singular points, if the cosmological constant is not zero. In the case of conformally coupled scalars, the number of singular points is reduced to four. In section three, we introduce the Schlesinger and Garnier systems which appear in the isomonodromy applications, and related the isomonodromic flow to the Painlevé VI system. In section four, we borrow from the theory of flat non-abelian connections to introduce a symplectic structure in the space of monodromy parameters. In section five, we consider the canonical transformation between the Painlevé VI system and the monodromy parameters. In section six, we discuss the implications for the scattering of eternal black holes, specifically between different asymptotic regions, and close 
with a summary of results. Some technical results about the monodromy of the hypergeometric equation and the asymptotics of the Painlevé system are presented in the appendix.

\section{Killing-Yano and separability}

A 2-form $h_{a b}$ is a principal conformal Killing-Yano tensor (PCKY) if it is a closed and non-degenerate conformal Killing-Yano tensor $(\mathrm{CKY})$, satisfying $\nabla_{(a} h_{b) c}=g_{a b} \eta_{c}-g_{c(a} \eta_{b)}$ for $\eta_{a}=\nabla^{b} h_{a b} /(D-1)$. Consider a spacetime $(M, g)$ with $D=2 n+\varepsilon$ dimensions allowing a PCKY, where $\varepsilon=0,1$. The existence of such structure implies a tower of $n-1$ KillingYano tensors, which implies $n$ Killing tensors if we include the metric tensor. Those killing tensors can then be used to construct $n+\varepsilon$ commuting killing vectors. Thus a spacetime with a PCKY has $D=2 n+\varepsilon$ conserved quantities. This is sufficient for integration of the geodesic equation [12], but it is also enough for complete separability of Klein-Gordon, Dirac and gravitational perturbation equations [13, 14].

Following [13], we can choose canonical coordinates $\left\{\psi_{i}, x_{\mu}\right\}$, where $\psi_{0}$ is the time coordinate, $\psi_{k}, k=1, \ldots, n-1+\epsilon$, are azimuthal coordinates - Killing vector affine parameters, and $x_{\mu}, \mu=1, \ldots, n$ stand for radial and latitude coordinates. In such coordinates the generic metric of $(M, g)$ which allows for a PCKY can be written as

$$
d s^{2}=\sum_{\mu=1}^{n}\left[\frac{d x_{\mu}^{2}}{Q_{\mu}}+Q_{\mu}\left(\sum_{k=0}^{n-1} A_{\mu}^{(k)} d \psi_{k}\right)^{2}-\frac{\epsilon c}{A^{(n)}}\left(\sum_{k=0}^{n-1} A^{(k)} d \psi_{k}\right)^{2}\right]
$$

where

$$
\begin{aligned}
Q_{\mu} & =\frac{X_{\mu}}{U_{\mu}}, \quad A_{\mu}^{(j)}=\sum_{\substack{\nu_{1}<\cdots<\nu_{j} \\
\nu_{i} \neq \mu}} x_{\nu_{1}}^{2} \ldots x_{\nu_{j}}^{2}, \quad A^{(j)}=\sum_{\nu_{1}<\cdots<\nu_{j}} x_{\nu_{1}}^{2} \ldots x_{\nu_{j}}^{2}, \\
U_{\mu} & =\prod_{\nu \neq \mu}\left(x_{\nu}^{2}-x_{\mu}^{2}\right), \quad X_{\mu}=\sum_{k=\epsilon}^{n} c_{k} x_{\mu}^{2 k}-2 b_{\mu} x_{\mu}^{1-\epsilon}+\frac{\epsilon c}{x_{\mu}^{2}}
\end{aligned}
$$

The polynomial $X_{\mu}$ is obtained by substituting the metric (2.1) into the $D$-dimensional Einstein equations. The metric with proper signature is recovered when we set $r=-i x_{n}$ and the mass parameter $M=(-i)^{1+\epsilon} b_{n}$.

One of the most interesting properties of the Kerr-NUT-(A)dS metric is separability. Consider the massive Klein-Gordon equation

$$
\left(\square-m^{2}\right) \Phi=0,
$$

its solution can be decomposed as

$$
\Phi=\prod_{\mu=1}^{n} R_{\mu}\left(x_{\mu}\right) \prod_{k=0}^{n+\epsilon-1} e^{i \Psi_{k} \psi_{k}}
$$

and substitution in (2.4) gives

$$
\left(X_{\mu} R_{\mu}^{\prime}\right)^{\prime}+\epsilon \frac{X_{\mu}}{x_{\mu}} R_{\mu}^{\prime}+\left(V_{\mu}-\frac{W_{\mu}^{2}}{X_{\mu}}\right) R_{\mu}=0,
$$


where

$$
W_{\mu}=\sum_{k=0}^{n+\epsilon-1} \Psi_{k}\left(-x_{\mu}^{2}\right)^{n-1-k}, \quad V_{\mu}=\sum_{k=0}^{n+\epsilon-1} \kappa_{k}\left(-x_{\mu}^{2}\right)^{n-1-k},
$$

and $\kappa_{k}$ and $\Psi_{k}$ are separation constants. For more details, see [14].

We shall focus in the $D=4$ case for the rest of the paper. In this case, we choose coordinates $\left(x_{1}, x_{2}, \psi_{0}, \psi_{1}\right)$, where $x^{\mu}, \mu=1,2$, represent the PCKY eigenvalues and $\psi_{i}$, $i=0,1$, are the Killing parameters of the 2 associated Killing vectors. Now if we set $\left(x_{1}, x_{2}, \psi_{0}, \psi_{1}\right) \equiv(p, i r, t, \phi)$, the metric $(2.1)$ is written as

$$
\begin{aligned}
d s^{2}= & \frac{r^{2}+p^{2}}{P(p)} d p^{2}+\frac{r^{2}+p^{2}}{Q(r)} d r^{2} \\
& +\frac{P(p)}{r^{2}+p^{2}}\left(d t-r^{2} d \phi\right)^{2} \\
& -\frac{Q(r)}{r^{2}+p^{2}}\left(d t+p^{2} d \phi\right)^{2}
\end{aligned}
$$

where $P(p)$ and $Q(r)$ are $4^{\text {th }}$ order polynomials given by [15]

$$
\begin{aligned}
P(p) & =-\frac{\Lambda}{3} p^{4}-\epsilon p^{2}+2 n p+k, \\
Q(r) & =-\frac{\Lambda}{3} r^{4}+\epsilon r^{2}-2 M r+k, \\
\epsilon=1-\left(a^{2}+6 b^{2}\right) \frac{\Lambda}{3}, \quad k & =\left(a^{2}-b^{2}\right)\left(1-b^{2} \Lambda\right), \quad n=b\left[1+\left(a^{2}-4 b^{2}\right) \frac{\Lambda}{3}\right] .
\end{aligned}
$$

The parameters are the black hole mass $M$, angular momentum to mass ratio $a$, cosmological constant $\Lambda$, and the NUT parameter $b$. To make contact with the physically meaningful Kerr-NUT-(A)dS metric, we set $p=b+a \cos \theta, \chi^{2}=1+\Lambda a^{2} / 3$, and make the substitution $\phi \rightarrow \phi / a \chi^{2}$ and $t \rightarrow\left(t-\frac{(a+b)^{2}}{a} \phi\right) / \chi^{2}$, in this order. If we set $b=0$ after this, we have the usual Kerr-(A)dS metric [16] in Chambers-Moss coordinates [15, 17, 18].

\section{$2.1 \quad$ Kerr-NUT-(A)dS case}

Let $\psi(t, \phi, r, \theta)=e^{-i \omega t} e^{i m \phi} R(r) S(\theta)$ be a solution of the Klein-Gordon equation for $D=4$ Kerr-NUT-(A)dS in Boyer-Lindquist coordinates. The radial equation resulting from this solution is

$$
\partial_{r}\left(Q(r) \partial_{r} R(r)\right)+\left(V_{r}(r)+\frac{W_{r}^{2}}{Q(r)}\right) R(r)=0
$$

where

$$
\begin{aligned}
Q(r) & =-\frac{\Lambda}{3} r^{4}+\epsilon r^{2}-2 M r+k, \\
\epsilon & =1-\left(a^{2}+6 b^{2}\right) \frac{\Lambda}{3}, \quad k=\left(a^{2}-b^{2}\right)\left(1-b^{2} \Lambda\right),
\end{aligned}
$$

and

$$
V_{r}=\kappa_{0} r^{2}+\kappa_{1}, \quad W_{r}=\Psi_{0} r^{2}+\Psi_{1},
$$




$$
\begin{array}{rlrl}
\kappa_{0} & =-4 \Lambda \xi, & \kappa_{1}=-C_{\ell}, \\
\Psi_{0}=\omega\left(1+\frac{\Lambda a^{2}}{3}\right), & \Psi_{1}=a\left(\omega \frac{(a+b)^{2}}{a}-m\right)\left(1+\frac{\Lambda a^{2}}{3}\right) .
\end{array}
$$

The parameter $\xi$ is the coupling constant between the scalar field and the Ricci scalar. Typical values of $\xi$ are minimal coupling, $\xi=0$, and conformal coupling, $\xi=1 / 6$. The separation constant between the angular and radial equations is $C_{\ell}$. The angular equation has essentially the same form as the radial one, associated to the problem of finding the eigenvalues of a second order differential operator with four regular singular points, in which case correspond to unphysical values for the latitude coordinates. The value of its eigenvalue $C_{\ell}$ can be approximated numerically from rational functions (Padé approximants), which were studied in this context by $[6,18]$. We refer to these authors for particular applications. For large values of the energy, it can be approximated by prolate spheroidal wave functions, whose behavior is tabulated.

In the following, we assume that all roots of $Q(r)$ are distinct and there are two real roots at least. When $\Lambda \rightarrow 0$, two of those roots match the Kerr horizons $\left(r_{+}, r_{-}\right)$and the other two diverge, leaving us with an irregular singular point of index 1 at infinity (and, therefore, a confluent Heun equation [6]). The characteristic coefficients - solutions for the indicial equations - of the finite singularities $r_{i}$ are

$$
\rho_{k}^{ \pm}= \pm i\left(\frac{\Psi_{0} r_{k}^{2}+\Psi_{1}}{Q^{\prime}\left(r_{k}\right)}\right), \quad k=1, \ldots, 4
$$

and for $r=\infty$ we have

$$
\rho_{\infty}^{ \pm}=\frac{3}{2} \pm \frac{1}{2} \sqrt{9-48 \xi}
$$

These coefficients give the local asymptotic behaviour of waves approaching any of the singular points, for example, one of the black hole horizons.

In this form, equation (2.10) has 5 regular singular points, including the point at infinity. It is possible to show that the point at infinity is actually an apparent singularity when $\xi=1 / 6$ and can be further removed by a gauge transformation. In that case, $(2.10)$ can be cast into a Heun type equation with 4 regular singular points given by the roots of $Q(r)=$ $-\frac{\Lambda}{3} \prod_{k=1}^{4}\left(r-r_{k}\right)$. This is done in the next section. A similar result has been reported by [19] for massless perturbations of spin $s=0, \frac{1}{2}, 1, \frac{3}{2}, 2$ for Kerr-(A)dS (the so-called Teukolsky master equation) and for $s=0, \frac{1}{2}$ for Kerr-Newman-(A)dS. One can show that Teukolsky master equation reduces to conformally coupled Klein-Gordon equation for scalar perturbations, being those perturbations of the Weyl tensor. Our computation below show this for the spin zero case, because of the explicit non-minimal coupling, and can be straightforwardly extended for higher spin cases. The reduction to Heun has also been shown true for $s=\frac{1}{2}, 1,2$ perturbations of all type-D metrics with cosmological constant [20].

\subsection{Heun equation from conformally coupled Kerr-NUT-(A)dS}

For $\xi=1 / 6$, it is possible to transform (2.10) with 5 regular singular points into a Heun equation with only 4 regular points. This is because $r=\infty$ in (2.10) becomes a removable singularity. In this section, we apply the transformations used in [20] for a scalar 
field, adapting the notation for our purposes, ${ }^{1}$ and we also calculate the difference between characteristic exponents, $\theta_{k}$, for each canonical form we obtain. As it turns out, these exponents are more useful to us because they are invariant under homographic and homotopic transformations [21], which preserve the monodromy properties of an ODE, as will be seen in section 4 .

By making the homographic transformation

$$
z=\frac{r-r_{1}}{r-r_{4}} \frac{r_{2}-r_{4}}{r_{2}-r_{1}}
$$

we map the singular points as

$$
\left(r_{1}, r_{2}, r_{3}, r_{4}, \infty\right) \quad \mapsto \quad\left(0,1, t_{0}, \infty, z_{\infty}\right)
$$

with

$$
z_{\infty}=\frac{r_{2}-r_{4}}{r_{2}-r_{1}}, \quad t_{0}=\frac{r_{3}-r_{1}}{r_{3}-r_{4}} z_{\infty} .
$$

Typically we set the relevant points for the scattering problem to $z=0$ and $z=1$, but we can consistently choose any two points to study. We note at this point that, for the de Sitter case, $t_{0}$ is a real number, which can be taken to be between 1 and $\infty$, whereas for the anti-de Sitter case, it is a pure phase $\left|t_{0}\right|=1$. Now, we define

$$
\begin{gathered}
\sigma_{ \pm}(r) \equiv \Psi_{0} r \pm \Psi_{1}, \\
f(r) \equiv 4 \xi \Lambda r^{2}+C_{l},
\end{gathered}
$$

and

$$
d_{k}^{-1} \equiv-\frac{\Lambda}{3} \prod_{\substack{j=1 \\ j \neq k}}^{3}\left(r_{k}-r_{j}\right)=\frac{Q^{\prime}\left(r_{k}\right)}{r_{k}-r_{4}} .
$$

Then, eq. (2.10) transforms to

$$
\begin{aligned}
& \frac{d^{2} R}{d z^{2}}+p(z) \frac{d R}{d z}+q(z) R=0 \\
& p(z)=\frac{1}{z}+\frac{1}{z-1}+\frac{1}{z-t_{0}}-\frac{2}{z-z_{\infty}} \\
& q(z)=\frac{F_{1}}{z^{2}}+\frac{F_{2}}{(z-1)^{2}}+\frac{F_{3}}{\left(z-t_{0}\right)^{2}}+\frac{12 \xi}{\left(z-z_{\infty}\right)^{2}}+\frac{E_{1}}{z}+\frac{E_{2}}{z-1}+\frac{E_{3}}{z-t_{0}}+\frac{E_{\infty}}{z-z_{\infty}},
\end{aligned}
$$

where

$$
F_{k}=\left(\frac{d_{k} \sigma_{+}\left(r_{k}^{2}\right)}{r_{k}-r_{4}}\right)^{2}=\left(\frac{\Psi_{0} r_{k}^{2}+\Psi_{1}}{Q^{\prime}\left(r_{k}\right)}\right)^{2},
$$

\footnotetext{
${ }^{1}$ Notice that [20] does not refer explicitly to the scalar case in their paper. However, our eq. (2.10) with $\xi=1 / 6$ can be obtained just by setting $s=0$ in eq. (11) of [20]. With respect to the parameters of [20], we must set $a=0$ and, in a non-trivial change, their term $2 g_{4} w^{2}$ must be equated to $-4 \Lambda \xi r^{2}$ to obtain the non-minimally coupled case.
} 


$$
\begin{aligned}
E_{\infty} & =\frac{12 \xi}{z_{\infty}\left(r_{4}-r_{1}\right)}\left(\sum_{k=1}^{3} r_{k}-r_{4}\right), \\
E_{k} & =\frac{d_{k}}{z_{k}-z_{\infty}}\left\{f\left(r_{k}\right)-\frac{2 \Lambda}{3} \frac{d_{k}^{2}}{r_{k}-r_{4}} \sigma_{+}\left(r_{k}^{2}\right)\left[\sigma_{-}\left(r_{k}^{2}\right) \sum_{j \neq k}^{3} r_{j}-2 r_{k} \sigma_{-}\left(\prod_{j \neq k}^{3} r_{j}\right)\right]\right\} .
\end{aligned}
$$

The $\theta_{k}$ for the finite singularities $z_{k}=\left\{0,1, t_{0}\right\}$ can be obtained by plugging $R(z) \sim$ $\left(z-z_{k}\right)^{\theta_{k} / 2}$ into $(2.21)$

$$
\theta_{k}=2 \sqrt{-F_{k}}=2 i\left(\frac{\Psi_{0} r_{k}^{2}+\Psi_{1}}{Q^{\prime}\left(r_{k}\right)}\right), \quad(k=1,2,3)
$$

and for the singularity at $z=\infty$,

$$
\begin{aligned}
\theta_{\infty} & =2 i \sqrt{12 \xi+E_{2}+t_{0} E_{3}+z_{\infty} E_{\infty}-\sum_{i=1}^{3} \frac{\theta_{i}^{2}}{4}} \\
& =2 i\left(\frac{\Psi_{0} r_{4}^{2}+\Psi_{1}}{Q^{\prime}\left(r_{4}\right)}\right),
\end{aligned}
$$

where the last equality follows from the invariance of $\theta$ under homographic transformations. It is possible to show that $\theta_{\infty}$ does not depend on $C_{\ell}$. Finally, for $z=z_{\infty}$ we have

$$
\theta_{z_{\infty}}=\sqrt{9-48 \xi}
$$

When the difference of any two characteristic exponents is an integer, we have a resonant singularity. This happens in (2.25) for $\xi=\{0,5 / 48,1 / 6,3 / 16\}$. Thus, we have a logarithmic behaviour near $z_{\infty}$, except for $\xi=1 / 6$ because, in this case, it is also a removable singularity, as will be seen briefly. The property of being removable only happens if $\theta_{z_{\infty}}$ is an integer different from zero. If $\theta_{z_{\infty}}=0$, we always have a logarithmic singularity. For more on this subject, see $[10,21,22]$.

To finish this section, we now show that (2.21) can be transformed into a Heun equation when $\xi=1 / 6$. First, we make the homotopic transformation

$$
R(z)=z^{-\theta_{0} / 2}(z-1)^{-\theta_{1} / 2}\left(z-t_{0}\right)^{-\theta_{t} / 2}\left(z-z_{\infty}\right)^{\beta} y(z) .
$$

The transformed ODE is now given by

$$
\frac{d^{2} y}{d z^{2}}+\hat{p}(z) \frac{d y}{d z}+\hat{q}(z) y=0
$$

where

$$
\begin{aligned}
& \hat{p}(z)=\frac{1-\theta_{0}}{z}+\frac{1-\theta_{1}}{z-1}+\frac{1-\theta_{t}}{z-t_{0}}+\frac{2 \beta-2}{z-z_{\infty}}, \\
& \hat{q}(z)=\frac{\hat{E}_{1}}{z}+\frac{\hat{E}_{2}}{z-1}+\frac{\hat{E}_{3}}{z-t_{0}}+\frac{\hat{E}_{\infty}}{z-z_{\infty}}+\frac{\hat{F}_{\infty}}{\left(z-z_{\infty}\right)^{2}},
\end{aligned}
$$


with

$$
\begin{aligned}
& \hat{E}_{k}=\frac{d_{k}}{z_{k}-z_{\infty}} f\left(r_{k}\right)+\sum_{j \neq k}^{3} \frac{\theta_{k}+\theta_{j}}{2\left(z_{j}-z_{k}\right)}+\frac{\theta_{k}(1-\beta)+\beta}{z_{k}-z_{\infty}}, \\
& \hat{E}_{\infty}=\frac{12 \xi}{z_{\infty}\left(r_{4}-r_{1}\right)}\left(\sum_{k=1}^{3} r_{k}-r_{4}\right)-\sum_{k=1}^{3} \frac{\theta_{k}(1-\beta)+\beta}{z_{k}-z_{\infty}}, \\
& \hat{F}_{\infty}=\beta^{2}-3 \beta+12 \xi
\end{aligned}
$$

Note that $\hat{F}_{\infty}=0$ is the indicial polynomial associated with the expansion at $z=z_{\infty}$. Thus it is natural to choose $\beta$ to be one of the characteristic exponents setting $\hat{F}_{\infty}=0$. However, to completely remove $z=z_{\infty}$ from (2.27), we need that $\beta=1$ in (2.28). This further constraints $\xi=1 / 6$ because of $(2.30 \mathrm{c})$. Now, we still need to check that $\hat{E}_{\infty}$ can be set to zero. The coefficients $\hat{E}$ above are simplified by noticing that

$$
\begin{aligned}
\sum_{k=1}^{4} \theta_{k} & =0, \quad \sum_{k=1}^{4} \theta_{k} r_{k}=\frac{6 i \Psi_{0}}{\Lambda} \\
\sum_{j \neq k}^{3} \frac{\theta_{k}+\theta_{j}}{2\left(z_{j}-z_{k}\right)} & =-2 i\left(\frac{d_{k}}{z_{k}-z_{\infty}}\right)\left(\frac{\Psi_{0} r_{4} r_{k}+\Psi_{1}}{r_{k}-r_{4}}\right), \quad(k=1,2,3)
\end{aligned}
$$

where we used the residue theorem to show these identities. This implies that, for $\beta=1$ and $\xi=1 / 6$,

$$
\begin{aligned}
\hat{E}_{k} & =\frac{d_{k}}{z_{k}-z_{\infty}}\left[f\left(r_{k}\right)-2 i\left(\frac{\Psi_{0} r_{4} r_{k}+\Psi_{1}}{r_{k}-r_{4}}\right)\right]+\frac{1}{z_{k}-z_{\infty}}, \\
\hat{E}_{\infty} & =\frac{1}{\left(r_{4}-r_{1}\right) z_{\infty}} \sum_{k=1}^{4} r_{k} .
\end{aligned}
$$

The polynomial $(2.9 \mathrm{~b})$ has no third-order term, so this means that the sum of all of its roots is zero. Therefore, $\hat{E}_{\infty}=0$ generically if $\beta=1$. This completes our proof that $(2.27)$ is a Fuchsian equation with 4 regular singular points, also called Heun equation.

Summing up, the radial equation of conformally coupled scalar perturbations of KerrNUT-(A)dS black hole can be cast as a Heun equation in canonical form

$$
y^{\prime \prime}+\left(\frac{1-\theta_{0}}{z}+\frac{1-\theta_{1}}{z-1}+\frac{1-\theta_{t_{0}}}{z-t_{0}}\right) y^{\prime}+\left(\frac{\kappa_{1} \kappa_{2}}{z(z-1)}-\frac{t_{0}\left(t_{0}-1\right) K_{0}}{z(z-1)\left(z-t_{0}\right)}\right) y=0,
$$

with coefficients

$$
\begin{aligned}
\theta_{k} & =2 i\left(\frac{\Psi_{0} r_{k}^{2}+\Psi_{1}}{Q^{\prime}\left(r_{k}\right)}\right), & k & =1,2,3,4, \\
K_{0} & =-\hat{E}_{3}, & t_{0} & =\frac{r_{3}-r_{1}}{r_{3}-r_{4}} \frac{r_{2}-r_{4}}{r_{2}-r_{1}},
\end{aligned}
$$


where we make the correspondence $k \in\{1,2,3,4\} \sim\left\{0,1, t_{0}, \infty\right\}$. The values of $\theta_{k}$ obey Fuchs relation, fixing $\kappa_{1,2}$ via $\theta_{0}+\theta_{1}+\theta_{t_{0}}+\kappa_{1}+\kappa_{2}=2$ and $\kappa_{2}-\kappa_{1}=\theta_{\infty}$. Also, in terms of (2.33) we have that $\kappa_{1} \kappa_{2}=\hat{E}_{2}+t_{0} \hat{E}_{3}=1+\theta_{4}$. These follow from the regularity condition at infinity, $\sum_{i=1}^{3} \hat{E}_{i}=0$. The set of 7 parameters $\left(\theta_{0}, \theta_{1}, \theta_{t_{0}}, \kappa_{1}, \kappa_{2} ; t_{0}, K_{0}\right)$ define the Heun equation and its fundamental solutions. By Fuchs relation, we see that the minimal defining set has 6 parameters. For more details about Heun equation, we refer to [21, 23].

In the Kerr-NUT-(A)dS case, we note the importance of $K_{0}$ indexing the solutions because the only dependence on $C_{\ell}$ comes from it. As mentioned before, the local Frobenius behaviour of the solutions do not depend on $C_{\ell}$, but this dependence will come about in the parametrization of the monodromy group done below.

The appearance of the extra singularity $t_{0}$ in (2.35) makes things more complicated than the hypergeometric case. First, the coefficients of the series solution obey a threeterm recurrence relation [23], which is not easily tractable to find explicit solutions [24]. Second, there is no known integral representation of Heun functions in terms of elementary functions, which hinders a direct treatment of the monodromies. Therefore, we need to look for an alternative approach to solve the connection problem of Heun equation. In the next sections, we will use the isomonodromic deformation theory [25-27] to shed light on this problem.

\section{Scattering, isomonodromy and Painlevé VI}

Scattering problems typically involve the calculation of a change of basis matrix between ingoing and outgoing Frobenius solutions of two singular points of an ordinary differential equation. This is the connection problem of a Fuchsian differential equation, as pointed out by Riemann and Poincaré. Fuchsian equations with 3 regular singular points have their connection problem solved, since the solutions are known to be expressed in terms of Gauss' hypergeometric function. For 4 regular points or more, the problem is still open. One alternative approach to the direct computation is the study of the symmetries - the integrable structure - of such systems. These go by the name of isomonodromic deformations [10, 25-27]. For 4 regular singular points, these are known to reduce to the study of Painlevé transcendents, and many results about the latter came about from the study of this integrable structure [28-30]. In the following sections we outline the application of these techniques to solve the scattering of scalar fields around black holes.

Linear ordinary differential equations like (2.35) are of Fuchsian type because their singular points $\{0,1, t, \infty\}$ are regular: the solution behaves as $y_{ \pm}(z) \approx\left(z-z_{i}\right)^{\rho_{i}^{ \pm}}$near a singular point $z_{i}$ and then its monodromy around each singular point is well known. By considering a solution of either type, we have that $y_{ \pm}\left(e^{2 \pi i}\left(z-z_{i}\right)\right)=e^{2 \pi i \rho_{i}^{ \pm}} y_{ \pm}(z)$. In the following, we suppose that $\rho^{ \pm}$are different, finite, and non-zero complex numbers whose difference is not an integer. The most natural setup to study monodromies are Fuchsian systems because, as mentioned in the introduction, the number of parameters defining them match the number of parameters of monodromy representations. Any Fuchsian equation can be written as a linear Fuchsian system with an appropriate gauge connection $A(z)$,

$$
\partial_{z} \mathcal{Y}(z)=A(z) \mathcal{Y}(z)
$$


where $\mathcal{Y}(z)$ is a column vector of two functions $y_{1}(z)$ and $y_{2}(z)[10]$. Now, let

$$
A(z)=\left(\begin{array}{ll}
A_{11}(z) & A_{12}(z) \\
A_{21}(z) & A_{22}(z)
\end{array}\right) .
$$

It can be verified that $y_{1}(z)$ satisfies the equation

$$
y^{\prime \prime}-\left(\frac{A_{12}^{\prime}}{A_{12}}+\operatorname{Tr} A(z)\right) y^{\prime}+\left(\operatorname{det} A(z)-A_{11}^{\prime}+A_{11} \frac{A_{12}^{\prime}}{A_{12}}\right) y=0,
$$

with a similar equation for $y_{2}(z)$. Now, if we are given a fundamental matrix of solutions $\Phi(z)=\left(\mathcal{Y}_{1}(z), \mathcal{Y}_{2}(z)\right)^{T}$, we can write the connection in terms of it

$$
A(z)=\left[\partial_{z} \Phi(z)\right] \Phi^{-1}(z),
$$

which tells us that $A=A(z) d z$ can be seen as a "pure gauge" $G L(2, \mathbb{C})$ gauge field, satisfying $F=d A+A \wedge A=0$. Since we are working in the $n$-punctured Riemann sphere, we are free to consider gauge transformations $\Phi(z) \rightarrow U(z) \Phi(z)$, or analogously, $A(z) \rightarrow U(z) A(z) U^{-1}(z)+\partial_{z} U(z) U^{-1}(z)$, where $U(z)$ has meromorphic functions for entries. These meromorphic functions can introduce apparent singularities, in which the indicial equation of (3.3) has integer values, and there is no logarithmic branching point. In this case the monodromy matrix around the apparent singularity is trivial: any composition of loops enclosing apparent singularities will have no effect on the monodromy associated with the loop.

As it turns out, an apparent singularity is exactly what one has in (3.3) when $A_{12}$ vanishes. Let $t=\left(t_{1}, t_{2}, \ldots, t_{n+3}\right)$ represent a set of $n+3$ singular points on the Riemann sphere, including $t_{n+1}=0, t_{n+2}=1$ and $t_{n+3}=\infty$, and let $\lambda=\left(\lambda_{1}, \lambda_{2}, \ldots, \lambda_{n}\right)$ represent the zeros of $A_{12}$. A Fuchsian system of Schlesinger type is written in a gauge where $A(z)$ has a partial fraction expansion

$$
A(z, t) d z=\sum_{i=1}^{n+2} \frac{A^{i}(t)}{z-t_{i}} d z
$$

where $A^{i}$ are matricial coefficients depending only on $t$. We can now ask if there is a way to change the positions of the regular singular points $t$ keeping the monodromies of (3.1) invariant. For that matter, we introduce the auxiliary system

$$
\partial_{t} \mathcal{Y}(z, t)=B(z, t) \mathcal{Y}(z, t) .
$$

As it turns out, in the Schlesinger gauge,

$$
B(z, t) d t=-\sum_{i=1}^{n} \frac{A^{i}(t)}{z-t_{i}} d t_{i},
$$

and the integrability condition for the Pfaffian system formed by (3.1) and (3.6) is given by the so called Schlesinger's equations $[9,10]$

$$
\frac{\partial A^{i}}{\partial t_{j}}=\frac{\left[A^{i}, A^{j}\right]}{t_{i}-t_{j}}, \quad j \neq i \quad \text { and } \quad \frac{\partial A^{i}}{\partial t_{i}}=-\sum_{j \neq i} \frac{\left[A^{i}, A^{j}\right]}{t_{i}-t_{j}} .
$$


The isomonodromy flow generated by the Schlesinger system above is Hamiltonian and has been studied extensively in a series of papers by Jimbo, Miwa and collaborators [25-27]. For the case of interest, the Heun equation, the phase space is two-dimensional, as we see below. We will use the asymptotics of the isomonodromy flow in order to solve for the monodromy problem of (2.35).

To better clarify the last paragraph and make contact with the monodromy problem of (2.35), we need to understand how the isomonodromic flow act on (3.3) in the case $n=1$. Let us choose a gauge where $\operatorname{Tr} A^{i}=\theta_{i}$ and where the off-diagonal terms of $A$ decay as $z^{-2}$ as $z \rightarrow \infty$. Then $A_{12}(z, t)$ has a single zero at $z=\lambda$, and is of the form

$$
A_{12}(z, t)=\frac{k(\lambda-z)}{z(z-1)(z-t)} .
$$

We fix the asymptotic behavior

$$
A^{\infty}=-\left(A^{0}+A^{1}+A^{t}\right)=\left(\begin{array}{cc}
\kappa_{1} & 0 \\
0 & \kappa_{2}-1
\end{array}\right),
$$

with $\kappa_{1}+\kappa_{2}=1-\theta_{0}-\theta_{1}-\theta_{t}$ and $\kappa_{2}-\kappa_{1}=\theta_{\infty}$ related to the parameters of the singular points. This choice introduces an extra singularity in (3.3). Plugging (3.2) with (3.9) into (3.3), we find a Fuchsian differential equation of Garnier type

$$
\begin{aligned}
& y^{\prime \prime}+p(z, t) y^{\prime}+q(z, t) y=0, \\
& p(z, t)=\frac{1-\theta_{0}}{z}+\frac{1-\theta_{1}}{z-1}+\frac{1-\theta_{t}}{z-t}-\frac{1}{z-\lambda}, \\
& q(z, t)=\frac{\kappa_{1} \kappa_{2}}{z(z-1)}-\frac{t(t-1) K}{z(z-1)(z-t)}+\frac{\lambda(\lambda-1) \mu}{z(z-1)(z-\lambda)} .
\end{aligned}
$$

The parameters $K$ and $\mu$ will play a significant role in the following and are related to $A(z)$ by

$$
\begin{aligned}
\mu= & \frac{A_{11}^{0}}{\lambda}+\frac{A_{11}^{1}}{\lambda-1}+\frac{A_{11}^{t}}{\lambda-t}, \\
K= & \frac{A_{11}^{t}}{\lambda-t}+\frac{A_{11}^{0}+A_{11}^{t}-\theta_{0} \theta_{t}}{t}+\frac{A_{11}^{1}+A_{11}^{t}-\theta_{1} \theta_{t}}{t-1} \\
& +\frac{1}{t} \operatorname{Tr} A^{0} A^{t}+\frac{1}{t-1} \operatorname{Tr} A^{1} A^{t} .
\end{aligned}
$$

In order to ensure that the singularity at $z=\lambda$ is apparent, $K$ is constrained to be a specific rational function of $\mu, \lambda$ and $t$,

$$
K(\lambda, \mu, t)=\frac{\lambda(\lambda-1)(\lambda-t)}{t(t-1)}\left[\mu^{2}-\left(\frac{\theta_{0}}{\lambda}+\frac{\theta_{1}}{\lambda-1}+\frac{\theta_{t}-1}{\lambda-t}\right) \mu+\frac{\kappa_{1} \kappa_{2}}{\lambda(\lambda-1)}\right] .
$$

The interesting thing about writing (3.11) in this form is that $K$ is a hamiltonian generating its isomonodromic flow in terms of $(\lambda(t), \mu(t))$. The isomodromic flow shuffles around 
the position of the apparent singularity $\lambda$ and the "conjugate momentum" $\mu$ in such a way to keep the monodromies fixed. Specifically, a change in the position of the true singularity $t$ entails a change in the parameters given by the Garnier system

$$
\frac{d \lambda}{d t}=\{K, \lambda\}, \quad \frac{d \mu}{d t}=\{K, \mu\}
$$

where the Poisson bracket is defined by

$$
\{f, g\}=\frac{\partial f}{\partial \mu} \frac{\partial g}{\partial \lambda}-\frac{\partial f}{\partial \lambda} \frac{\partial g}{\partial \mu} .
$$

The Schlesinger equations (3.8) are given in this case by (see, for instance, [31]):

$$
\frac{d A^{0}}{d t}=\frac{\left[A^{t}, A^{0}\right]}{t}, \quad \frac{d A^{1}}{d t}=\frac{\left[A^{t}, A^{1}\right]}{t-1}, \quad \frac{d A^{t}}{d t}=\frac{\left[A^{0}, A^{t}\right]}{t}+\frac{\left[A^{1}, A^{t}\right]}{t-1} .
$$

The Hamiltonian associated with the Schlesinger system is $t(t-1) H=(t-1) \operatorname{Tr} A^{0} A^{t}+$ $t \operatorname{Tr} A^{1} A^{t}$, which is the term in the second line of the expression for $K$ above. The two Hamiltonians $K$ and $H$ are thus related by a canonical transformation [10, 29]. Since the entries $A_{11}^{0}, A_{11}^{1}$ and $A_{11}^{t}$ can be explicitly computed in terms of $\mu, \lambda, t$ the Garnier and the Schlesinger systems are actually equivalent. Explicit expressions can be found in $[10,26]$.

Our conclusion is that $\mu$ and $\lambda$ are canonically conjugated coordinates in the phase space of isomonodromic deformations. If we write the equation of motion in terms of $\lambda$ alone,

$$
\begin{aligned}
\ddot{\lambda}= & \frac{1}{2}\left(\frac{1}{\lambda}+\frac{1}{\lambda-1}+\frac{1}{\lambda-t}\right) \dot{\lambda}^{2}-\left(\frac{1}{t}+\frac{1}{t-1}+\frac{1}{\lambda-t}\right) \dot{\lambda} \\
& +\frac{\lambda(\lambda-1)(\lambda-t)}{2 t^{2}(1-t)^{2}}\left(\theta_{\infty}^{2}-\theta_{0}^{2} \frac{t}{\lambda^{2}}+\theta_{1}^{2} \frac{t-1}{(\lambda-1)^{2}}+\left(1-\theta_{t}^{2}\right) \frac{t(t-1)}{(\lambda-t)^{2}}\right),
\end{aligned}
$$

which corresponds to the sixth Painlevé equation $P_{V I}$. This is the more general second order differential equation of the form $\ddot{z}=R(z, \dot{z}, t)$, with $R$ a rational function, which has the Painlevé property: the singularities of $\lambda(t)$, apart from $t=0,1, \infty$, are simple poles and depend on the choice of initial conditions. Given a particular set of initial conditions, the equation can then be used to define a new transcendental function, the Painlevé transcendent $\mathcal{P}_{V I}\left(\theta_{\infty}, \theta_{0}, \theta_{1}, \theta_{t} ; t\right)$, in the same way the linear second order ordinary equation with 3 regular singular points can be used to define the hypergeometric function [10,30].

Now we see how the theory of isomonodromic deformations can help us to solve our initial scattering problem: Painlevé VI asymptotics are given in terms of the monodromy data of (3.11). In section 5, we show how to relate (3.11) with (2.35) and how Painlevé asymptotics solve the monodromy problem of Heun equation. But first, in the next section, we make a mathematical digression about how to parameterize the monodromy group of Fuchsian systems.

\section{Flat connections and monodromies}

Physically, the formulation in terms of the flat connection (3.4) with the decomposition (3.5) means that the scattering problem is equivalent to finding the potential of a holomorphic 
$G L(2, \mathbb{C})$ Yang-Mills field with a number of monopoles with non-abelian charges $A^{i}[32]$. It is then reasonable to expect that, given the positions of the charges and their coefficients, the monodromy values will be uniquely defined.

Mathematically, the space of such flat connections, $\mathcal{A}_{g, n}$, is associated with the moduli space of genus $g$ Riemann surfaces with $n$ punctures $\mathcal{M}_{g, n}$, and our case of interest will be the Riemann sphere where $g=0$. Because of global conformal transformations,

$$
z \rightarrow \tilde{z}=\frac{a z+b}{c z+d}, \quad \tilde{A}(\tilde{z}) d \tilde{z}=A(z) d z,
$$

we are able to fix 3 of the $n$ singular points and $\mathcal{M}_{g, n}$ is thus covered by the anharmonic ratios like

$$
\frac{\left(z-z_{n}\right)\left(z_{n-1}-z_{n-2}\right)}{\left(z-z_{n-2}\right)\left(z_{n-1}-z_{n}\right)}
$$

which allowed us to write Heun equation (2.35) in terms of the position of a single pole $t_{0}$ alone. The other independent parameter appearing in (2.35) is $K_{0}$. In order to describe it geometrically, let us consider a generic Fuchsian equation in the normal form with $n$ finite singular points, that is

$$
\begin{gathered}
\psi^{\prime \prime}(w)+T(w) \psi(w)=0, \quad T(w)=\sum_{i=1}^{n}\left(\frac{\delta_{i}}{\left(w-w_{i}\right)^{2}}+\frac{c_{i}}{w-w_{i}}\right), \\
\sum_{i=1}^{n} c_{i}=0, \quad \sum_{i=1}^{n}\left(c_{i} w_{i}+\delta_{i}\right)=0, \quad \sum_{i=1}^{n}\left(c_{i} w_{i}^{2}+2 \delta_{i} w_{i}\right)=0,
\end{gathered}
$$

where (4.4) are the necessary and sufficient conditions for $w=\infty$ to be a regular point. Because of (4.4), there are only $n-3$ independent $c_{i}$, and we can also fix 3 of the $z_{i}$ to be 0,1 and $\infty$ by a homographic transformation. Thus, if we fix the $\delta_{i}$, we can parametrize Fuchsian equations by $2(n-3)$ complex numbers $\left(c_{i}, z_{i}\right)$. Typically, we say that $\delta_{i}$ and $z_{i}$ are local parameters, depending only on local behaviour of solutions, and the $c_{i}$, usually called accessory parameters, have global properties not probed locally. The accessory parameters are usually related to spectral parameters of differential equations [21, 23]. We notice now that the angular eigenvalue $C_{l}$ dependence appears exactly in the accessory parameter of Heun equation, and that is why it did not appear in the Frobenius coefficients $\theta_{i}$.

We can relate (4.3) to our Heun equation (2.35) by setting $n=4$ and applying a homographic transformation such that $\left(w_{1}, w_{2}, w_{3}, w_{4}, \infty ; w\right) \mapsto\left(0,1, t, \infty, z_{\infty} ; z\right)$. We also need to remove the apparent singularity by letting $\psi \mapsto\left(z-z_{\infty}\right)^{-1} \psi$, finally giving us the equation

$$
\psi^{\prime \prime}(z)+\tilde{T}(z) \psi(z)=0, \quad \tilde{T}(z)=\sum_{i=1}^{3}\left(\frac{\delta_{i}}{\left(z-z_{i}\right)^{2}}+\frac{\tilde{c}_{i}}{z-z_{i}}\right)
$$

such that

$$
\tilde{c}_{i}=\frac{c_{i}\left(w_{4}-w_{i}\right)-2 \delta_{i}}{z_{i}-z_{\infty}}, \quad \sum_{i=1}^{3} \tilde{c}_{i}=0
$$


Note that

$$
\sum_{i=1}^{3} \frac{c_{i} w_{4 i}^{2}-2 \delta_{i} w_{4 i}}{z-z_{i}}=\frac{\tilde{c}_{2}+t \tilde{c}_{3}}{z(z-1)}+\frac{t(t-1) \tilde{c}_{3}}{z(z-1)(z-t)} .
$$

Analyzing the behaviour of (4.5) at infinity, we may rewrite $\tilde{c}_{2}+t \tilde{c}_{3}$ as $\delta_{4}-\left(\delta_{1}+\delta_{2}+\delta_{3}\right)$, reproducing the conventions used in [33]. Finally, we now take (2.35) and make $y(z)=$ $N(z) \psi(z)$, where

$$
N(z)=\exp \left(-\frac{1}{2} \int \hat{p}(z) d z\right)=\prod_{i=1}^{3}\left(z-z_{i}\right)^{-\left(1-\theta_{i}\right) / 2},
$$

to obtain (4.5). Within this transformation, we can check that

$$
\tilde{T}(z)=\hat{q}(z)-\frac{\hat{p}^{2}(z)}{4}-\frac{\hat{p}^{\prime}(z)}{2}
$$

which implies $\delta_{i}=\left(1-\theta_{i}^{2}\right) / 4$ and also

$$
\tilde{c}_{i}=\frac{1-d_{i} f\left(r_{i}\right)}{z_{i}-z_{\infty}}+\sum_{j \neq i}^{3} \frac{\left(1+\theta_{i} \theta_{j}\right)}{2 z_{j i}} .
$$

One of the most important results of the mathematical investigation in [32] is that the space of flat connections $\mathcal{A}_{0, n}$ has a natural symplectic form $\Omega$. It basically stems from the fact that flat connections have a natural action, the Chern-Simons form, living in a space with an extra dimension, apart from the coordinates $z, \bar{z}$, with the extra dimension interpreted as a gauge parameter:

$$
\delta S=\int_{\Sigma \times \mathbb{C}} \operatorname{Tr}(\delta A \wedge F)+2 \int_{\Sigma} \operatorname{Tr}(\delta A \wedge A)
$$

Thus one relates the variation of the boundary " $p \delta q$ " term, $\frac{1}{2 \pi i} \operatorname{Tr}(\delta A \wedge \delta A)$, to the AtiyahBott symplectic form $\Omega$. The construction is reminiscent of the appearance of the so-called Wess-Zumino term in WZW models in conformal field theory. As it turns out [34, 35], the $n-3$ independent accessory parameters and the $n-3$ independent poles $z_{i}$ are a set of Darboux coordinates for $\Omega$, that is:

$$
\Omega=\sum_{i}^{n-3} d c_{i} \wedge d z_{i}
$$

Specializing to the four singularity case, we have that, in terms of the canonical form of the equation (2.35), the symplectic form can be readily written in terms of the position of the singularities and the "Hamiltonian":

$$
\Omega=d K \wedge d t,
$$

as can be anticipated from the Hamiltonian form of the Painlevé equation. One has a heuristical correspondence between Heun's equation and Painlevé VI: the latter can be 
understood as the classical hamiltonian system (3.15), whereas Heun's equation is obtained from $K$ if we treat it as the "quantum hamiltonian". In fact, from (3.14) one has:

$$
K\left(z,-\frac{\partial}{\partial z}, t\right) y(z)=0
$$

as the Heun equation, modulo an integer shift of the $\theta_{i} \cdot{ }^{2}$ Thus, in a sense, the Painlevé VI equation is the classical limit of the Heun equation [36]. This also reinforces the view that the position of the singular point $t$ and the accessory parameter $K$ should be seen as conjugate quantities.

Incidentally, the other five equations from the Painlevé list can be obtained from the sixth by a scaling limit (confluence). An object of further study is whether this allows for calculation of monodromies in the confluent Heun case.

The flow means that the "phase space" $\left\{z_{n}, c_{n}\right\}$ can be foliated into integral curves of the Hamiltonian (3.14). The monodromy data is constant over these curves, so they are effectively functions of the space of orbits. We want, however, to get the monodromy data from the values of $K$ and $t$. In order to do this, we will have to review the algebraic aspects of the monodromy matrices.

The Fuchsian equation is defined in the Riemann sphere $\mathbb{C P}^{1} \sim \mathbb{C} \cup\{\infty\}$ minus $n$ points $\left\{z_{1}, z_{2}, \ldots, z_{n}\right\}$ in which the fundamental matrix of solutions diverge like $\left(z-z_{i}\right)^{\rho_{i}^{ \pm}}$with $\rho_{i}^{ \pm}$the solutions of the indicial equation. Following the usual RiemannHilbert problem formulation [37], we will define the fundamental group of such space with a fixed point $z_{0}$, and construct a representation as follows. Let $\gamma_{i}$ be a curve containing $z_{0}$ that divides the punctured sphere into two regions, one containing only $z_{i}$ and the other containing all other singular points. We associate with $\gamma_{i}$ a matrix $M_{i}$ which mixes the two solutions of the general ODE (2.10). Clearly

$$
M_{1} M_{2} \ldots M_{n}=\mathbb{I}
$$

since the composition of all $\gamma_{i}$ is a contractible curve. The famous Riemann-Hilbert problem consists in finding an ODE with a given set of monodromy data $M_{i}$. Our problem is quite the opposite: how to determine $M_{i}$ from the data readily available in the ODE.

Of course the problem does not have a single solution, if one finds a particular set of matrices $\left\{M_{i}\right\}$ satisfying (4.15), then $\left\{g M_{i} g^{-1}\right\}$ will also be a solution, corresponding to a diferent choice of fundamental solutions. Also, the indicial equation allows us to write the solution near a singular point: up to a change of basis, the monodromy matrix near a regular singular point is:

$$
M_{i}=\Lambda_{i}^{-1} \exp \left[\rho_{i} \mathbb{I}+\alpha_{i} \sigma^{3}\right] \Lambda_{i}, \quad \rho_{i}^{ \pm}=\rho_{i}^{0} \pm \alpha_{i}, \quad \Lambda_{i} \in G L(2, \mathbb{C}),
$$

so the conjugacy class of each $M_{i} \in G L(2, \mathbb{C})$ is known. In the following we will assume without loss of generality that $\rho_{i}^{0}=0$, which reduces the group to $\operatorname{SL}(2, \mathbb{C})$. For the

\footnotetext{
${ }^{2}$ This was called a Schlesinger transformation in [25-27]. Note that the integer shift doesn't change monodromies around a single singular point.
} 
application to ODEs these are set by the Fuchs relation. Let us define $g_{i}$ as the matrix that changes basis between the fiducial point $z_{0}$ and the $z_{i}$ :

$$
M_{i}=g_{i}^{-1} \exp \left[\alpha_{i} \sigma^{3}\right] g_{i}, \quad \alpha_{i}=i \pi \theta_{i} .
$$

The determination of the $g_{i}$ is important for computing scattering elements. Given a "purely ingoing" or "purely outgoing" solution near $z_{i}$, the scattering amplitudes from another point $z_{j}$ are given by

$$
\mathcal{M}_{i \rightarrow j}=g_{i} g_{j}^{-1}=\left(\begin{array}{cc}
1 / \mathcal{T} & \mathcal{R} / \mathcal{T} \\
\mathcal{R}^{*} / \mathcal{T}^{*} & 1 / \mathcal{T}^{*}
\end{array}\right)
$$

where $\mathcal{T}$ and $\mathcal{R}$ are the transmission and reflection amplitudes, respectively [6].

We can now turn back to the problem of relating the monodromy data to the accessory parameters in (2.35). We will review the construction outlined in [35]. Let $M_{i}$ be the monodromy matrix as above. The monodromy data readily available from the differential equation are the traces:

$$
m_{i}=\operatorname{Tr}\left(M_{i}\right), \quad i=0,1, t . \quad \text { and } \quad m_{\infty}=\operatorname{Tr}\left(M_{0} M_{1} M_{t}\right)=\operatorname{Tr}\left(M_{\infty}^{-1}\right) .
$$

In order to fully characterize the $M_{i}$ (up to an overall conjugation), we need the other characters:

$$
m_{01}=\operatorname{Tr}\left(M_{0} M_{1}\right), \quad m_{0 t}=\operatorname{Tr}\left(M_{0} M_{t}\right), \quad m_{1 t}=\operatorname{Tr}\left(M_{1} M_{t}\right) .
$$

The set of $m$ 's are not all independent, they satisfy the Fricke-Jimbo relation:

$$
\begin{aligned}
W\left(m_{0 t}, m_{1 t}, m_{01}\right)= & m_{0 t} m_{1 t} m_{01}+m_{0 t}^{2}+m_{1 t}^{2}+m_{01}^{2}-m_{0 t}\left(m_{1} m_{\infty}+m_{0} m_{t}\right) \\
& -m_{1 t}\left(m_{0} m_{\infty}+m_{1} m_{t}\right)-m_{01}\left(m_{t} m_{\infty}+m_{0} m_{1}\right) \\
& +m_{0}^{2}+m_{1}^{2}+m_{t}^{2}+m_{\infty}^{2}+m_{0} m_{1} m_{t} m_{\infty}-4=0,
\end{aligned}
$$

which gives a quadratic relation that allow one to compute one of the $m_{i j}$, say, $m_{1 t}$, given the other two, $m_{01}$ and $m_{0 t}$. The configuration space for the monodromy data with fixed $m_{i}$ and $m_{\infty}$ is then parametrized by 2 independent variables. We can give the solution for the monodromy matrices parametrized by the set of $m_{i}$ and $m_{i j}$ : given a Euler-angle parametrization of the $g_{i}$ 's:

$$
g_{i}=\exp \left[\psi_{i} \sigma^{3} / 2\right] \exp \left[\phi_{i} \sigma^{1} / 2\right] \exp \left[\varphi_{i} \sigma^{3} / 2\right]
$$

one notes immediately that $\varphi_{i}$ can all be set to zero, while the parametrization of the $m_{i j}$ can be verified by simple matrix multiplication:

$$
m_{i j}=2 \cosh \alpha_{i} \cosh \alpha_{j}+2 \sinh \alpha_{i} \sinh \alpha_{j}\left(\cosh \phi_{i} \cosh \phi_{j}+\sinh \phi_{i} \sinh \phi_{j} \cosh \left(\psi_{i}-\psi_{j}\right)\right) \text {, }
$$

and $m_{\infty}$ given by the Fricke-Jimbo relation. One notes that there is an overall symmetry $\psi_{i} \rightarrow \psi_{i}+\psi$, which can be used to reduce the overall number of parameters to 5 , in the Heun 

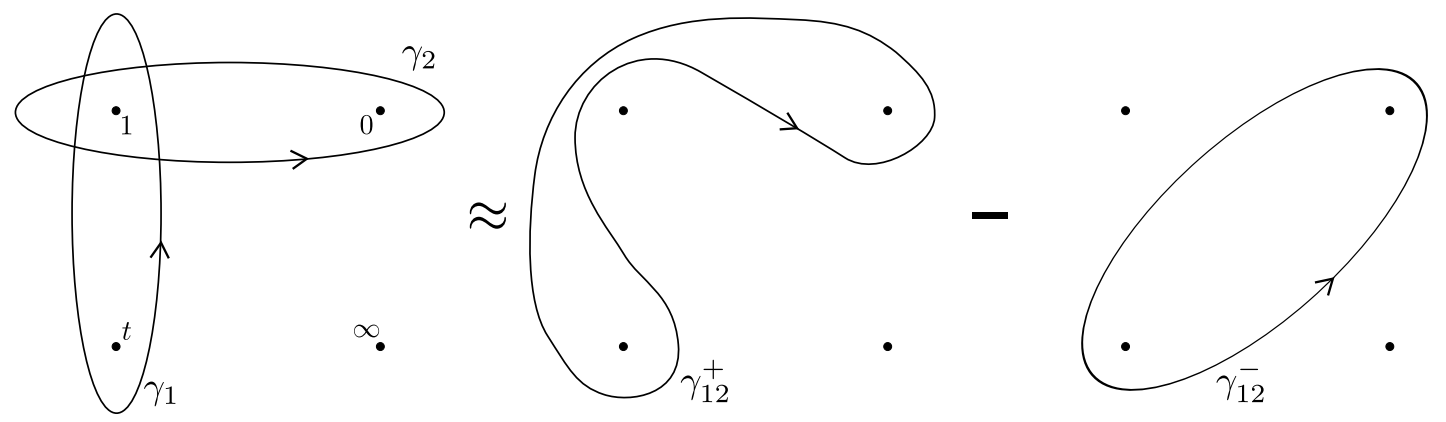

Figure 1. The skein-relation applied to the four-punctured sphere. In the left hand side we represent the Poisson bracket by drawing both curves simultaneously.

case. We are given the four $\theta_{i}$, so there is one unfixed free parameter in the monodromy matrices which depends explicitly on the accessory parameter $K$ and the anharmonic ratio $t$.

With the $\theta_{i}$ fixed, the set of two complex numbers $m_{01}$ and $m_{0 t}$ provide a local set of coordinates to the space of flat connections $\mathcal{A}_{0,4}$. These coordinates are not canonical, in the sense we will explore now.

As stated in the preceding section, $\mathcal{A}_{g, n}$ has a natural symplectic structure, given by the Atiyah-Bott formula:

$$
\Omega=\frac{1}{2 \pi i} \int_{\Sigma} \operatorname{Tr}(\delta A \wedge \delta A) .
$$

The traces of the monodromies are formally given by the Wilson loops:

$$
m_{\gamma}=\operatorname{Tr} M_{\gamma}=\operatorname{Tr} \mathcal{P} \exp \left[\oint_{\gamma} A(z) d z\right]
$$

Using (4.24) one can compute the skein-relations, relating different holonomies [38]:

$$
\left\{m_{\gamma_{i}}, m_{\gamma_{j}}\right\}=\frac{1}{2} \sum_{x \in \gamma_{i} \cap \gamma_{j}}\left(m_{\gamma_{x, i, j}^{+}}-m_{\gamma_{x, i, j}^{-}}\right),
$$

where the loops $\gamma_{x, i, j}^{ \pm}$are constructed by removing a small neighborhood of the intersection point $x$ and replacing it by two arcs. The superscript labels the two choices of completion. See figure 1.

In terms of the variables $m_{01}, m_{0 t}$ and $m_{1 t}$, the skein relation implies:

$$
\left\{m_{1 t}, m_{01}\right\}=\rho^{+}-m_{0 t}
$$

where, because of the Cayley-Hamilton theorem, we have for an unimodular matrix $\operatorname{Tr}(A) \mathbb{I}=A+A^{-1}$, and then:

$$
\rho^{+}=\operatorname{Tr}\left(M_{1}^{-1} M_{t} M_{1} M_{0}\right)=-m_{0 t}-m_{1 t} m_{01}+m_{0} m_{t}+m_{1} m_{\infty} .
$$

Note also that the Poisson bracket is related to the function $W$ defined by (4.21):

$$
\left\{m_{1 t}, m_{01}\right\}=-\frac{\partial W}{\partial m_{0 t}} .
$$


Given this relation, one can now introduce canonically conjugated coordinates $\phi$ and $\psi$ on $\mathcal{M}_{0,4}$, so that the monodromies $m_{i j}$ are parametrized as follows:

$$
\begin{aligned}
m_{1 t} & =2 \cos \pi \phi \\
m_{01} & =\frac{2 \cos \pi \psi}{m_{1 t}^{2}-4} \sqrt{c_{1 t} c_{0 \infty}}-2 \frac{\left(m_{0} m_{1}+m_{t} m_{\infty}\right)-\cosh \pi \phi\left(m_{0} m_{t}+m_{1} m_{\infty}\right)}{m_{1 t}^{2}-4} \\
m_{0 t} & =\frac{\sin \pi \phi}{2 \sin \pi \psi} \sqrt{c_{1 t} c_{0 \infty}}-\frac{1}{2}\left(m_{1 t} m_{01}-m_{0} m_{t}-m_{1} m_{\infty}\right)
\end{aligned}
$$

with

$$
c_{1 t}=m_{1 t}^{2}+m_{1}^{2}+m_{t}^{2}-m_{1 t} m_{0} m_{1}-4, \quad c_{0 \infty}=m_{1 t}^{2}+m_{0}^{2}+m_{\infty}^{2}-m_{1 t} m_{0} m_{\infty}-4 .
$$

Given that $\phi$ and $\psi$ are independent Darboux coordinates, we have, up to a multiplicative constant,

$$
\Omega=d \phi \wedge d \psi
$$

and then the transformation from the parameters in the Heun equation $(2.35)(t, K)$ to the monodromy parametrization $(\phi, \psi)$ is canonical.

\section{The classical mechanics of monodromies}

Now we turn to the problem of finding the canonical transformation that takes the parameters of the Heun equation to the monodromy parametrization $\phi$ and $\psi$. By canonical, one means that there exists a function $f$ such that

$$
K \equiv K(\phi, t)=\frac{\partial}{\partial t} f(\phi, t)
$$

with $f(\phi, t)$ the generating function of the transformation. This function has been receiving some attention recently because its relation to conformal blocks in Liouville field theory [39, 40]. For recent developments both in the application for Liouville and $c=1$ conformal blocks see $[7,33,41-43]$. There it appears as the semiclassical approximation to the 5-point function of conformal primaries. It is the WKB approximation to the Ward identity:

$$
\left[\frac{1}{b^{2}} \frac{\partial^{2}}{\partial z^{2}}+\sum_{n=1}^{4}\left(\frac{\Delta_{i}}{\left(z-z_{i}\right)^{2}}+\frac{1}{z-z_{i}} \frac{\partial}{\partial z}\right)\right]\left\langle V_{(1,2)}(z) V_{\Delta_{1}}\left(z_{1}\right) \ldots V_{\Delta_{4}}\left(z_{4}\right)\right\rangle=0
$$

up to contact terms. Because of conformal invariance, this expectation value also depends only on the anharmonic ratios between the coordinates, $\left\{t_{i}\right\}$, and the classical limit yields:

$$
\left\langle V_{(1,2)}(z) V_{\Delta_{1}}\left(z_{1}\right) \ldots V_{\Delta_{4}}\left(z_{4}\right)\right\rangle_{b \rightarrow 0}=\psi(z, t) \exp \left(\frac{1}{b^{2}} f(\phi, t)\right) .
$$

Where $\psi(z, t)$ is a solution of the Heun equation in the normal form and $f(\phi, t)$ is as in (5.1). For more details about Liouville correlators and the Riemann-Hilbert problem see [44].

Now the equations of motion for isomonodromic transformations is obvious in terms of the variables $\phi$ and $\psi$ : they are constant. Therefore, the generating function of the canonical transformation is the action itself, calculated at a solution of the Painlevé equation [45]. 
To wit, let us remind that, since we are dealing with canonically conjugate coordinates, we can write the action 1 -form $\alpha$ using either pair:

$$
\alpha=\mu d \lambda-K(\mu, \lambda, t) d t=\psi d \phi-H(\phi, \psi, S) d S .
$$

The Hamiltonian with respect to the $\phi$ and $\psi$ coordinates is trivial, since the flow is isomonodromic. We will take $H=-1$ and identify $S$ with the action computed on the solutions of the equations of motion:

$$
d S=\mu d \lambda-K(\mu, \lambda, t) d t-\psi d \phi
$$

Hence

$$
\mu=\frac{\partial S}{\partial \lambda}, \quad \psi=-\frac{\partial S}{\partial \phi}, \quad K=-\frac{\partial S}{\partial t} .
$$

That is, $S$ is a function of the independent variables $\lambda, t$ and $\phi$. Observing the last equality, we can then invert and have the monodromy as a function of $K$ and $t$ :

$$
K=-\frac{\partial S}{\partial t}(\lambda, \phi, t) \Rightarrow \phi=\phi(K, \lambda, t)
$$

Now, if the system is computed at the solutions of the equation of motion, $\lambda(t)$ satisfies the Painlevé VI and $\mu(t)$ is given by

$$
\dot{\lambda}=\frac{\partial K}{\partial \mu}=\frac{\lambda(\lambda-1)(\lambda-t)}{t(t-1)}\left[2 \mu-\left(\frac{\theta_{0}}{\lambda}+\frac{\theta_{1}}{\lambda-1}+\frac{\theta_{t}-1}{\lambda-t}\right)\right] .
$$

Given that $\phi$ is constant over the solutions, this leads to

$$
S(\phi, \lambda, t)=\int_{\left(\lambda_{i}, t_{i}\right)}^{(\lambda, t)} \mu\left(\lambda, t^{\prime}\right) d \lambda-K\left(\mu\left(\lambda, t^{\prime}\right), \lambda, t^{\prime}\right) d t^{\prime} .
$$

The path of integration $(\lambda(s), s)$ is a solution of the Painlevé VI equation with initial condition given by $\lambda_{i}, t_{i}$ and monodromy parameter given by $\phi$. The dependence on $\phi$ has been considered in a number of papers $[28,46]$, and is explicit at the Painlevé singular points $t=0,1, \infty$. Let us take $t_{i} \rightarrow 1$ as the asymptotic point for definiteness. One should note that we can always take this to be the case by a permutation of the singular points of the Heun equation - whose action in the Painlevé equation is known as the bi-rational transformation [29]. Near the singular point, we assume further that $0<\operatorname{Re} \phi<1$, so one can find that (see appendix A)

$$
\lambda(t)=1+\kappa(\phi, \psi)(1-t)^{1-\phi}+\ldots, \quad \mu(t)=\frac{1}{2 \kappa(\phi, \psi)}\left(\theta_{1}+\theta_{t}-\phi\right)(1-t)^{-1+\phi}+\ldots,
$$

where $\kappa(\phi, \psi)$ is a complicated, but known (A.21), function of the monodromies, and the ellipses denote subdominant terms in the assumption $0<\operatorname{Re} \phi<1$. Fixed this, the action is now a function of the condition at the upper limit of integration, which is the position of the apparent singularity $\lambda$ and the real singularity at $t$.

Now, the upper limit of the integration can also be fixed by the application we have in mind. The Heun equation has four singular regular points, whereas the Garnier system has 
five regular points, where one of them, at $\lambda$ is an apparent singularity. We can then envision a condition which the apparent singularity coincides with one of the other singular points, say $t$, and the value of $\theta_{t}$ is shifted by one. The condition $\lambda\left(t_{0}\right)=t_{0}$ seems then natural, and to fix the values for $K\left(t_{0}\right)$ and $\mu\left(t_{0}\right)$ we consider the limit $\lambda \rightarrow t$ in (2.35). Then we have $K\left(t_{0}\right)-\mu\left(t_{0}\right)=K_{0}$ and by taking the same limit in the Hamiltonian (3.14), we find:

$$
\lambda\left(t_{0}\right)=t_{0}, \quad \mu\left(t_{0}\right)=-\frac{K_{0}}{\theta_{t}-1} .
$$

One can then study the asymptotics of the Painlevé system with these initial conditions and extract the values of $\phi$ and $\psi$ in the asymptotic limit $t \rightarrow 1$. We will leave the full numerical investigation to future work.

Instead, let us remind that the action $S$ in (5.9) is a solution of the Hamilton-Jacobi equation, and so it implements the canonical transformation between $\lambda$ and $\mu$ and the monodromy parameters $\phi$ and $\psi$. On the solutions of the equations of motion, the variations of the action only depend on the initial and final points of the trajectory. So,

$$
\psi=-\frac{\partial S}{\partial \phi}=-\left.\mu \frac{\partial \lambda}{\partial \phi}\right|_{1} ^{t_{0}}
$$

where the $t \rightarrow 1$ limit has to be taken with care. With the asymptotic conditions (5.10), the integral has the logarithmic divergence for $t \rightarrow 1$ :

$$
S=\frac{1}{4}\left(\left(\theta_{1}+\theta_{t}\right)^{2}-\phi^{2}\right) \log (1-t)+\ldots
$$

and this term will be subtracted from the form of the action in order for us to obtain a finite result for (5.12). One should point out that the ellipsis is not analytic at $t=1$, but still vanishes in the limit. Since the term subtracted is a function of $t$ alone, the regularized action will still be minimized by the solutions of the Painlevé and still solve the HamiltonJacobi equations. Similar comments were made in [33], although the end result above is better suited for the isomonodromy problem of the Garnier system.

The specifics of the system renders the usual tools used to study the isomonodromy problem less than perfect. For instance, one has the definition of the $\tau$-function for the Painlevé flow:

$$
\frac{d}{d t} \log \tau(t)=K(\lambda(t), \mu(t), t)
$$

where we use the convention in [10]. Other definitions, such as found in [26, 28, 31, 42] differ by an explicit function of time. From this definition one has the immediate interpretation of the $\tau$ function as (exponential of) the classical action for zero momentum configurations. These solutions are of importance to the theory of uniformization and to special solutions of Painlevé VI (see [42]). For the case at hand, however, one would like to introduce a "generalized" $\tau$-function, integrating the whole Lagrangean.

Finally, one sees from this theory a strange symmetry of the scattering process. Because the flow of $t$ is defined on the complex plane, we can always restrict the movement to the "real" submanifold where $t$ has a physical interpretation. We use the word "real" loosely here: for the anti-de Sitter case, we recall that $t_{0}$ is a phase. One can then consider a line 


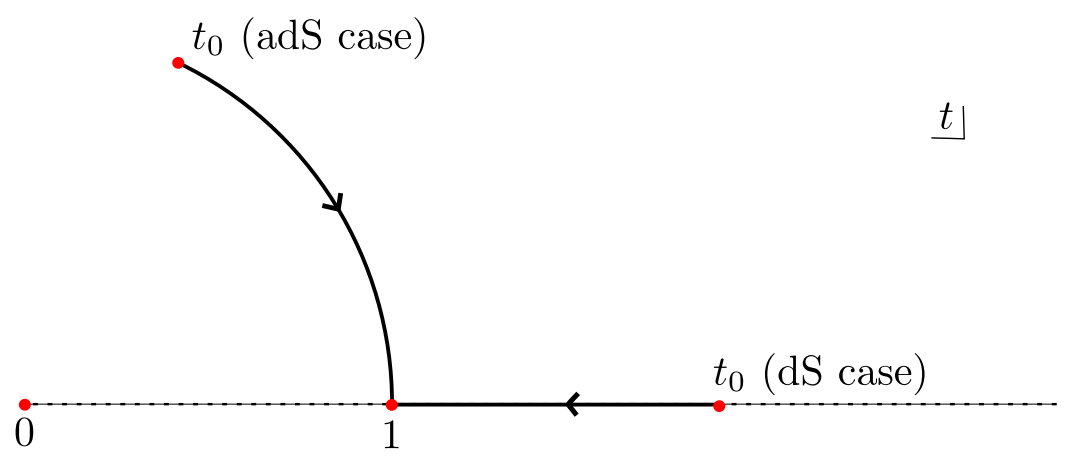

Figure 2. The "real" curves in the complex $t$ plane where the parameters have a physical interpretation. It is an arc of the unit circle for anti-de Sitte and a line segment for the de Sitter case.

of "real phases" linking $t_{0}$ to 1 , as in the figure 2 . For each point of, say, the arc linking $t_{0}$ to 1 , one has a pair of parameters $(t, K(t))$ with the same scattering properties as $\left(t_{0}, K_{0}\right)$. The values for $\lambda(t), \mu(t)$ won't matter for the scattering because the singularity at $\lambda$ is apparent. The de Sitter case is much the same, but now the line of physical parameters is also real. That this symmetry is deeply linked to the Painlevé transcendent is something of a surprise and surely its understanding deserves more work.

\section{The generic scattering}

In essence, the procedure outlined in the preceeding sections does give scattering elements in terms of the changing basis matrices $g_{i} g_{j}^{-1}$. Apart from computing transmission and reflecting coefficients to black hole solutions, the full set of monodromies can be used to compute scattering elements between different asymptotic regions.

We will be interested in the Kerr-AdS case where the real singularity points correspond to the horizons and spatial infinity, and all singular points are regular. Usually, one is interested in the region $r_{+}<r<\infty$, where "classical" movement takes place. The interpretation of elements of $g_{i} g_{j}^{-1}$ as scattering elements (4.18) comes about because one chooses the purely "ingoing" solution at the singular point $r_{+}$, as in figure 4 . One then associates with the matrix an oriented path, or a graph, between the two singularities. The transmission and reflection coefficients should be seen as a linear map between the asymptotic region and itself, the S-matrix.

However, the black hole metric in general can be analytically continued past $r_{+}$. In terms of generic Kruskal coordinates, which follow geodesics, the region $r>r_{+}$is but one of many different asymptotically AdS regions. This is schematically presented through the Penrose diagram in figure 3. The metric for the other regions is obtained by analytic continuation: each corresponds to a different leaf of the Riemann surface determined by the solution of the Einstein equations.

In other words, the metric in the other asymptotic regions is obtained by analytic continuation "around" the singularity. In the Kerr-AdS case, as in many others, the form of the metric in the "inside" of the black hole, or, more appropriately, the regions II and III in figure 3 , is obtained by selecting $r_{-}<r<r_{+}$in the form of the metric. The passage between 


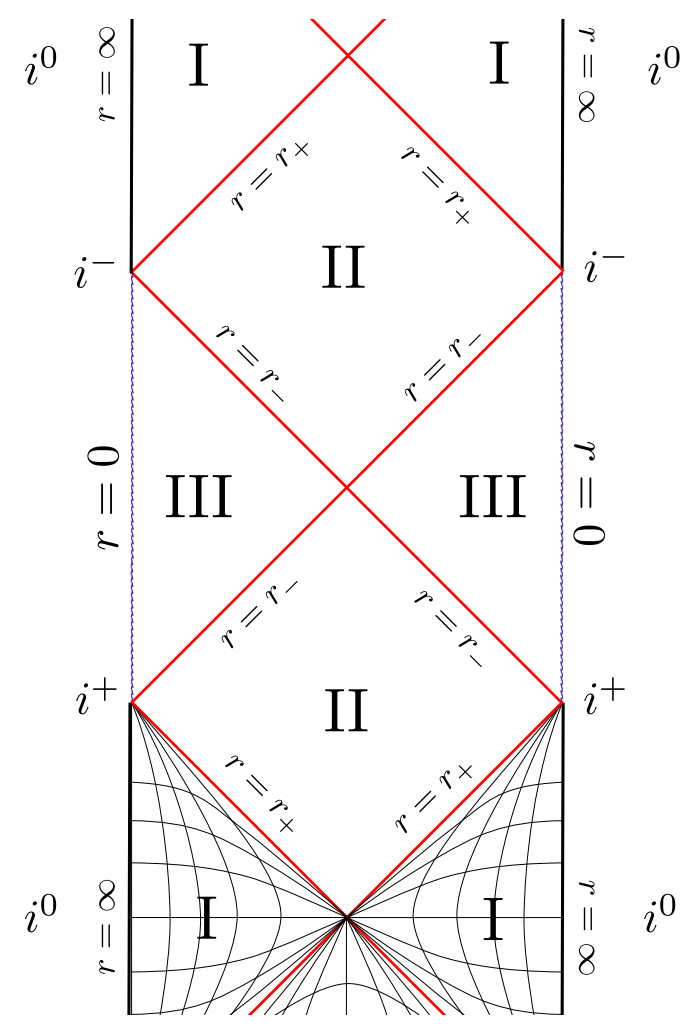

Figure 3. The causal (Penrose) diagram of the (eternal) Kerr black hole in AdS space. To each asymptotic region one assigns a Hilbert space $\mathcal{H}_{I}$.
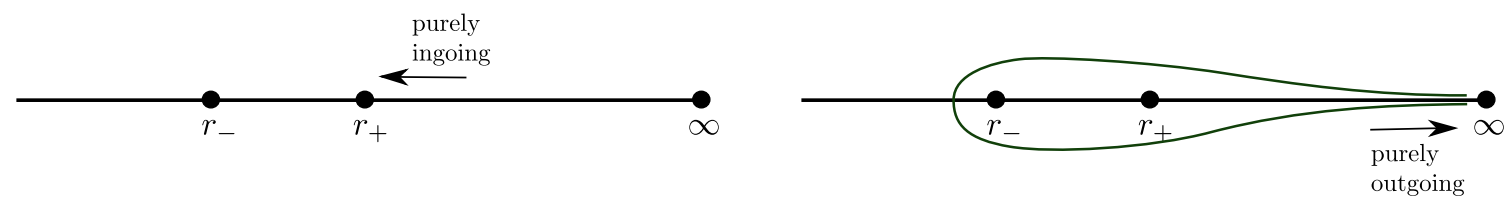

Figure 4. The schematics of scattering. In the left hand side, the constrain that the solution is "purely ingoing" at $r=r_{+}$. In the right-hand side, the monodromy associated with a solution that emerges at a different region $I$.

different coordinate patches are constructed from global, Kruskal-like coordinates, but the end result can be understood as analytical continuation around the singular points $r=r_{+}$ and $r=r_{-}$. Provided one keeps to the real line, the metric thus obtained will also be real and describe the other regions of the black hole. Generically, as we go around the complex $z$ plane back to the asymptotic region of large $z$, the solution of the Klein-Gordon equation will pick a monodromy. But, by the construction outlined, the large $z$ region will belong to some other asymptotically AdS region. This is illustrated in the right hand side of figure 4 .

How to interpret the monodromy matrix then? If one chooses a basis like pictured in figure 4, where one has a "purely outgoing" wave at spatial infinity, the monodromy path is naturally associated with the wave travelling along regions II and III in 3. Since the monodromy path cannot be disentangled, one should return to a different leaf of the Riemann surface parametrized by complex $r$. Physically, one returns to a different asymptotic region 
I. The monodromy matrix then allows us to compute the scattering coefficients between different asymptotic regions. As a result, one should assign to each asymptotic region I a Hilbert space $\mathcal{H}_{I}$. Theoretically, one could also assign a Hilbert space to the singularity region $r=0$, but it is less clear what is that the scattering coefficients are measuring, and we will omit this discussion in the following.

A similar construct was outlined before $[47,48]$ in other to describe local states in the interior of the black hole. There, as in here, one points out the necessity of the other asymptotic regions in order to describe the black hole interior. In [49], this type of scattering was used to probe the region near the singularity. In all of those discussions, the number of regions is limited to two, because one is dealing with Schwarzschild black holes. The causal diagram for black holes with zero angular momentum is singular in which there are only two separate asymptotic regions, as in the Kerr black hole. Taking generic lessons from the analysis of these singular cases may be dangerous.

The method outlined here should work for the generic case. In the case where one obtains a hypergeometric equation for the radial part, incidentally, corresponds to cases of extreme black holes where the extra asymptotic regions are also missing. This can be further verified by studing the extremal limit where $t_{0} \rightarrow 1$ in (2.35). Dealing with the Heun equation is paramount to study the phenomenon of scattering through the black hole. We hope to address this quantitatively in the future.

There is, however, some generic conclusions one can take from the monodromy method without resorting to numerical computations on the Painlevé transcendents. First and foremost, the isomonodromy flow points to a hidden symmetry of the Klein-Gordon equation, where different accessory parameters $K$ give off the same scattering elements. We suspect this can be better understood from twistor methods.

Secondly, the subgroup of $\mathrm{SL}(2, \mathbb{C})$ generated by $M_{i}$ will in general have no closed orbits. A Kleinian group [50] is defined as a discrete subgroup of $\operatorname{SL}(2, \mathbb{C})$. Classically, Kleinian groups appear as monodromies of algebraic solutions of the Heun equation, which, in turn, are related to crystallographic groups. One famous family of examples are the triangle groups of tesselations of the Poincaré disk. These appear in the special case where the conjugacy classes of the monodromy matrices are rational numbers (and purely imaginary).

When we are in the generic case where this doesn't happen, but still the traces $m_{i}$ are purely imaginary, the orbits of the group generated by the monodromies describe dense circles in the group. Generically, then, the amplitudes of processes interpolating between different asymptotic regions will interfere destructively and cancel out. Because of this, any process that has to be traced over an infinite number of asymptotic regions will appear to be unitary. Therefore, in order to measure the effects that those different asymptotic regions have in the scattering process, one has to go beyond quantum mechanics and study field theoretic (higher order) correlations. This sort of "black hole complementarity" stems from the attribution that different asymptotic regions should have independent Hilbert spaces. The question of locality in quantum mechanics is sufficiently muddled in order for this not to be a trivial assumption. At any rate, one sees no reason in order that effects from these regions should not play a significant role in field theoretical processes, where one goes beyond the two-point function evolution. 


\section{Discussion}

In this paper we discussed the application of the isomonodromy method to the calculation of scattering amplitudes of a generic Kerr-NUT-(A)dS black hole. These spacetimes have the necessary algebraic properties to ensure separability of the wave equation, which can be cast as the problem of two (coupled) Fuchsian ordinary differential equations. The algebraic property turns out to be the existence of a conformal Killing-Yano tensor, which is closely tied to Petrov type D spacetimes, and those are further tied to a subjacent twistorial structure. The fact that we had, for generic values for the curvature coupling $\xi$, mass $M$, angular momentum $a=J / M$, NUT charge $b$ and cosmological constant $\Lambda$, a Fuchsian equation is a remarkable fact in itself. We found further that, while the generic coupling has 5 regular singular points for the radial equation, ${ }^{3}$ the conformally coupled case $\xi=1 / 6$ has one apparent singularity, and the ensuing equation is of Heun type $[19,20]$.

Since the connection problem for the Heun equation is an open problem, we turned to the isomonodromy method. Although the method has historical ties to Fuchsian differential equations, it is more suited to linear Fuchsian systems, as discussed in section 3. The difference is that the latter has apparent singularities with trivial monodromies, with apparent singularities obeying the Painlevé property, and hence can be "integrated" in the sense that the ensuing differential equations define uniquely a function on the complex plane. The case of four singular points, the Heun case, results in the Painlevé VI equation for the dynamic of the position of the apparent singularity. Other cases (and other Painlevé equations) are obtained from confluence, and this is a very interesting problem in itself, deeply tied with the so-called Stokes phenomenon with applications in the scattering of Kerr black holes in flat spaces and the representation of the Virasoro algebra for Liouville field theory. This should be the object of future studies.

The application of the isomonodromy method to the connection problem of the Heun equation is somewhat simpler than the analogous problem of monodromy of the four regular singular point Schlesinger system. The guidelines of solving it where outlined in [33], using the Hamilton-Jacobi method described in section 4 and 5. Since the application for black hole scattering selects naturally an initial condition for the Painlevé system, the action needs only regularization at one particular point, which we chose to be $t=1$. There is a mathematical relation between the action $S$ and the $\tau$ function introduced in field theoretic applications [25], but for the generic black hole scattering it is an extension of the latter. The result can be obtained numerically and will be presented separately.

In section 6 we present a discussion of how the knowledge of the full monodromy problem can shed light on aspects of black hole complementarity, specially questions of unitarity and scattering between different asymptotic regions. Mathematically there seems to be a deep connection to the theta function associated to an isomonodromic flow [26], which can be used to detect deviations from the purely unitary scattering. This feature is particular to the Heun equation, and do not show in cases where it reduces to the hypergeometric case.

\footnotetext{
${ }^{3}$ This equation has been associated with the name of Böcher in classical treatises [51], which states that all equations of classical mathematical physics can be derived from it through the process of confluence. It is amusing that the same seems to hold for black hole scattering!
} 
Amusingly, the hypergeometric cases are obtained when the spacetime does not display multiple asymptotic regions, like in the extremal black hole and $A d S_{2} \times S^{2}$ cases. The study of the generic case of scattering will surely be of impact not only to black hole physics, but also to generic correlations in dual systems described in the gauge/gravity correspondence.

\section{Acknowledgments}

The authors would like to thank Amílcar de Queiroz, Dmitry Melnikov, Mark MineevWeinstein, A. Yu. Morozov and Marc Casals for useful discussions and comments. Fábio Novaes acknowledges partial support from CNPq.

\section{A Schlesinger system asymptotics and Painlevé VI}

Here we list the relevant results in the asymptotics of Painlevé VI as studied by [28] and [46] and listed in [52]. The problem was also considered in [53]. In order to study the monodromy near the point $t=0$, consider the Schlesinger equations for the Heun system (3.17). In the $t \rightarrow 0$ approximation we have

$$
\frac{d A^{0}}{d t}=\frac{\left[A^{t}, A^{0}\right]}{t}, \quad \frac{d A^{1}}{d t}=-\left[A^{t}, A^{1}\right], \quad \frac{d A^{t}}{d t} \approx \frac{\left[A^{0}, A^{t}\right]}{t}+\mathcal{O}\left(t^{0}\right) .
$$

This means that, near $t=0, A^{t}$ and $A^{0}$ have a logarithmic divergence

$$
A^{0} \approx t^{\Lambda} A_{0}^{0} t^{-\Lambda}, \quad \text { and } \quad A^{t} \approx t^{\Lambda} A_{0}^{t} t^{-\Lambda} \quad \text {, where } \Lambda=A_{0}^{0}+A_{0}^{t},
$$

whereas $A^{1}$ has a continuous limit as $t \rightarrow 0$. In terms of the fundamental matrix $\Phi(z, t)$ in (3.4), the system splits into an equation for $\Phi_{0}(z)=\lim _{t \rightarrow 0} \Phi(z, t)$ and another for $\Phi_{1}(z)=\lim _{t \rightarrow 0} t^{-\Lambda} \Phi(t z, t)$

$$
\frac{d \Phi_{0}}{d z}=\left(\frac{A_{0}^{1}}{z-1}+\frac{\Lambda}{z}\right) \Phi_{0}, \quad \frac{d \Phi_{1}}{d z}=\left(\frac{A_{0}^{0}}{z}+\frac{A_{1}^{t}}{z-1}\right) \Phi_{1}
$$

Each problem gives a hypergeometric connection. Assuming the general case where there is no integer difference between the exponents, the solutions are

$$
\begin{aligned}
& \Phi_{0}=\Phi\left(\frac{1}{2}\left(\theta_{\infty}-\theta_{1}-\phi\right) ;-\frac{1}{2}\left(\theta_{\infty}+\theta_{1}+\phi\right) ; 1-\phi ; z\right) z^{-\phi / 2}(z-1)^{-\theta_{1} / 2} \\
& \Phi_{1}=G_{1} \Phi\left(-\frac{1}{2}\left(\theta_{0}+\theta_{t}+\phi\right) ;-\frac{1}{2}\left(\theta_{0}+\theta_{t}-\phi\right) ; 1-\theta_{0} ; z\right) C_{1} z^{-\theta_{0} / 2}(z-1)^{-\theta_{t} / 2}
\end{aligned}
$$

with the hypergeometric fundamental solution given by

$$
\Phi(\alpha, \beta ; \gamma ; z)=\left(\begin{array}{ll}
\Phi_{11} & \Phi_{12} \\
\Phi_{21} & \Phi_{22}
\end{array}\right) z^{-\left(\begin{array}{cc}
\alpha & 0 \\
0 & \beta
\end{array}\right) .}
$$


and

$$
\begin{aligned}
& \Phi_{11}={ }_{2} F_{1}\left(\alpha, \alpha-\gamma+1 ; \alpha-\beta ; \frac{1}{z}\right), \quad \Phi_{22}={ }_{2} F_{1}\left(\beta, \beta-\gamma+1 ; \beta-\alpha ; \frac{1}{z}\right), \\
& \Phi_{12}=\frac{\beta(\beta-\gamma+1)}{(\beta-\alpha)(\beta-\alpha+1)} \frac{1}{z}{ }_{2} F_{1}\left(\beta+1, \beta-\gamma+2 ; \beta-\alpha+2 ; \frac{1}{z}\right), \\
& \Phi_{21}=\frac{\alpha(\alpha-\gamma+1)}{(\alpha-\beta)(\alpha-\beta+1)} \frac{1}{z}{ }_{2} F_{1}\left(\alpha+1, \alpha-\gamma+2 ; \alpha-\beta+2 ; \frac{1}{z}\right) .
\end{aligned}
$$

The constants $\alpha, \beta$ and $\gamma$ are given by

$$
\alpha=\frac{1}{2}\left(\theta_{\infty}-\theta_{1}-\phi\right), \quad \beta=\frac{1}{2}\left(-\theta_{\infty}-\theta_{1}-\phi\right), \quad \gamma=1-\phi .
$$

The asymptotics of the hypergeometrics are

$$
Y(\alpha, \beta, \gamma ; z)= \begin{cases}G_{\alpha \beta \gamma}^{(0)}(1+\mathcal{O}(z)) z\left(\begin{array}{rr}
-\gamma & 0 \\
0 & 0
\end{array}\right) C_{\alpha \beta \gamma}^{(0)}, & z \rightarrow 0, \\
G_{\alpha \beta \gamma}^{(1)}(1+\mathcal{O}(z-1))(z-1)\left(\begin{array}{rr}
\gamma-\alpha-\beta-1 & 0 \\
0 & 0
\end{array}\right) C_{\alpha \beta \gamma}^{(1)}, & z \rightarrow 1, \\
\left(1+\mathcal{O}\left(z^{-1}\right)\right) z\left(\begin{array}{cc}
-\alpha & 0 \\
0 & -\beta
\end{array}\right), & z \rightarrow \infty,\end{cases}
$$

where

$$
G_{\alpha \beta \gamma}^{(0)}=\frac{1}{\beta-\alpha}\left(\begin{array}{c}
\beta-\gamma+1 \\
\alpha-\gamma+1 \alpha
\end{array}\right), \quad G_{\alpha \beta \gamma}^{(1)}=\frac{1}{\beta-\alpha}\left(\begin{array}{c}
1 \beta(\beta-\gamma) \\
1 \alpha(\alpha-\gamma)
\end{array}\right),
$$

and the connection matrices are

$$
\begin{aligned}
& C_{\alpha \beta \gamma}^{(0)}=\left(\begin{array}{cc}
e^{-\pi i(\alpha-\gamma+1) \frac{\Gamma(\gamma-1) \Gamma(\alpha-\beta+1)}{\Gamma(\gamma-\beta) \Gamma(\alpha)}} & e^{-\pi i(\beta-\gamma+1) \frac{\Gamma(\gamma-1) \Gamma(\beta-\alpha+1)}{\Gamma(\gamma-\alpha) \Gamma(\beta)}} \\
e^{-\pi i \alpha \frac{\Gamma(1-\gamma) \Gamma(\alpha-\beta+1)}{\Gamma(1-\beta) \Gamma(\alpha-\gamma+1)}} & -e^{-\pi i \beta \frac{\Gamma(1-\gamma) \Gamma(\beta-\alpha+1)}{\Gamma(1-\alpha) \Gamma(\beta-\gamma+1)}}
\end{array}\right), \\
& C_{\alpha \beta \gamma}^{(1)}=\left(\begin{array}{cc}
-\frac{\Gamma(\alpha+\beta-\gamma+1) \Gamma(\alpha-\beta+1)}{\Gamma(\alpha-\gamma+1) \Gamma(\alpha)} & \frac{\Gamma(\alpha+\beta-\gamma+1) \Gamma(\beta-\alpha+1)}{\Gamma(\beta-\gamma+1) \Gamma(\beta)} \\
-e^{-\pi i(\gamma-\alpha-\beta-1) \Gamma(\gamma-\alpha-\beta-1) \Gamma(\alpha-\beta+1)} & e^{-\pi i(\gamma-\alpha-\beta-1) \Gamma(\gamma-\alpha-\beta-1) \Gamma(\beta-\alpha+1)}
\end{array}\right) .
\end{aligned}
$$

The asymptotics of the $A^{i}$ are worked out in [28], giving

$$
\begin{aligned}
\Lambda+\frac{1}{2} \phi \mathbb{I} & \simeq \frac{1}{4 \theta_{\infty}}\left(\begin{array}{cc}
\left(-\theta_{\infty}-\theta_{1}+\phi\right)\left(\theta_{\infty}-\theta_{1}-\phi\right) & \left(-\theta_{\infty}-\theta_{1}+\phi\right)\left(\theta_{\infty}+\theta_{1}+\phi\right) \\
\left(\theta_{\infty}-\theta_{1}+\phi\right)\left(\theta_{\infty}-\theta_{1}-\phi\right) & \left(\theta_{\infty}-\theta_{1}+\phi\right)\left(\theta_{\infty}+\theta_{1}+\phi\right)
\end{array}\right) \\
A_{1}^{0}+\frac{1}{2} \theta_{1} \mathbb{I} & \simeq \frac{1}{4 \theta_{\infty}}\left(\begin{array}{cc}
-\left(\theta_{\infty}-\theta_{1}\right)^{2}+\phi^{2} & \left(\theta_{\infty}+\theta_{1}\right)^{2}-\phi^{2} \\
-\left(\theta_{\infty}-\theta_{1}\right)^{2}+\phi^{2} & \left(\theta_{\infty}+\theta_{1}\right)^{2}-\phi^{2}
\end{array}\right) \\
A_{0}^{0}+\frac{1}{2} \theta_{0} \mathbb{I} & =G_{1} \frac{1}{4 \phi}\left(\begin{array}{cc}
\left(\theta_{0}-\theta_{t}+\phi\right)\left(\theta_{0}+\theta_{t}+\phi\right) & \left(\theta_{0}-\theta_{t}+\phi\right)\left(-\theta_{0}-\theta_{t}+\phi\right) \\
\left(\theta_{0}-\theta_{t}-\phi\right)\left(\theta_{0}+\theta_{t}+\phi\right) & \left(\theta_{0}-\theta_{t}-\phi\right)\left(-\theta_{0}-\theta_{t}+\phi\right)
\end{array}\right) G_{1}^{-1}
\end{aligned}
$$




$$
A_{t}^{0}+\frac{1}{2} \theta_{t} \mathbb{I}=G_{1} \frac{1}{4 \phi}\left(\begin{array}{c}
\left(\theta_{t}+\phi\right)^{2}-\theta_{0}-\left(\theta_{t}-\phi\right)^{2}+\theta_{0}^{2} \\
\left(\theta_{t}+\phi\right)^{2}-\theta_{0}-\left(\theta_{t}-\phi\right)^{2}+\theta_{0}^{2}
\end{array}\right) G_{1}^{-1}
$$

The matrix is given by

$$
G_{1}=G_{\alpha \beta \gamma}^{(0)}\left(\begin{array}{cc}
1 & 0 \\
0 & -\hat{s}^{-1}
\end{array}\right)
$$

with

$$
\begin{gathered}
\hat{s}=\frac{\Gamma(1-\phi)^{2} \Gamma\left(\frac{1}{2}\left(\theta_{0}+\theta_{t}+\phi\right)+1\right) \Gamma\left(\frac{1}{2}\left(-\theta_{0}+\theta_{t}+\phi\right)+1\right)}{\Gamma(1+\phi)^{2} \Gamma\left(\frac{1}{2}\left(\theta_{0}+\theta_{t}-\phi\right)+1\right) \Gamma\left(\frac{1}{2}\left(-\theta_{0}+\theta_{t}-\phi\right)+1\right)} \times \\
\times \frac{\Gamma\left(\frac{1}{2}\left(\theta_{\infty}+\theta_{1}+\phi\right)+1\right) \Gamma\left(\frac{1}{2}\left(-\theta_{\infty}+\theta_{1}+\phi\right)+1\right)}{\Gamma\left(\frac{1}{2}\left(\theta_{\infty}+\theta_{1}-\phi\right)+1\right) \Gamma\left(\frac{1}{2}\left(-\theta_{\infty}+\theta_{1}-\phi\right)+1\right)} s
\end{gathered}
$$

and the parameter $s$ given by

$$
\begin{aligned}
4 \sin & \frac{\pi}{2}\left(\theta_{0}+\theta_{t} \mp \phi\right) \sin \frac{\pi}{2}\left(\theta_{0}-\theta_{t} \pm \phi\right) \sin \frac{\pi}{2}\left(\theta_{\infty}+\theta_{1} \mp \phi\right) \sin \frac{\pi}{2}\left(\theta_{\infty}-\theta_{1} \pm \phi\right) s^{ \pm}= \\
= & \left( \pm i \sin \pi \phi \cos \pi \sigma_{1 t}-\cos \pi \theta_{t} \cos \pi \theta_{\infty}-\cos \pi \theta_{0} \cos \pi \theta_{1}\right) e^{ \pm \pi i \phi} \\
& \pm i \sin \pi \phi \cos \pi \sigma_{01}+\cos \pi \theta_{t} \cos \pi \theta_{1}+\cos \pi \theta_{\infty} \cos \pi \theta_{0} .
\end{aligned}
$$

With these expressions, one can calculate the asymptotic expansion for the $\tau$ function

$$
\frac{d}{d t}\left(t(t-1) \frac{d}{d t} \log \tau(t)\right)=\theta_{\infty} A_{22}^{t}-\frac{1}{2} \theta_{t}^{2}
$$

obtaining [28]

$$
\begin{aligned}
\tau(t) \simeq t^{\left(\phi^{2}-\theta_{0}^{2}-\theta_{t}^{2}\right) / 4}\left[1+\frac{1}{8 \phi^{2}}\left(\theta_{0}^{2}-\theta_{t}^{2}-\phi^{2}\right)\left(\theta_{\infty}^{2}-\theta_{1}^{2}-\phi^{2}\right) t\right. \\
\quad-\frac{\hat{s}}{16 \phi^{2}\left(1+\phi^{2}\right)}\left(\theta_{0}^{2}-\left(\theta_{t}-\phi\right)^{2}\right)\left(\theta_{\infty}^{2}-\left(\theta_{1}-\phi\right)^{2}\right) t^{1+\phi} \\
\left.\quad-\frac{\hat{s}^{-1}}{16 \phi^{2}\left(1-\phi^{2}\right)}\left(\theta_{0}^{2}-\left(\theta_{t}+\phi\right)^{2}\right)\left(\theta_{\infty}^{2}-\left(\theta_{1}+\phi\right)^{2}\right) t^{1-\phi}+\mathcal{O}\left(|t|^{2(1-\operatorname{Re} \phi)}\right)\right] .
\end{aligned}
$$

For the asymptotics as $t \rightarrow 1$, one just need to change $\theta_{0}$ to $\theta_{1}$, and $\lambda(t)$ to $\lambda(t)-1$. Finally, the asymptotic formula for the Painlevé transcendent itself, as in [52]:

$$
\lambda(t) \simeq 1+\frac{\left(\theta_{t}-\theta_{1}+\phi\right)\left(\theta_{t}+\theta_{1}+\phi\right)\left(\theta_{\infty}+\theta_{0}+\phi\right)}{4 \phi^{2}\left(\theta_{\infty}+\theta_{0}-\phi\right) \hat{s}}(1-t)^{1-\phi}\left(1+\mathcal{O}\left(t^{\phi}, t^{1-\phi}\right)\right),
$$

assuming, as always, $0<\operatorname{Re} \phi<1$.

Open Access. This article is distributed under the terms of the Creative Commons Attribution License (CC-BY 4.0), which permits any use, distribution and reproduction in any medium, provided the original author(s) and source are credited. 


\section{References}

[1] N.D. Birrell and P.C.W. Davies, Quantum fields in curved space, Cambridge University Press, Cambridge U.K. (1982).

[2] D.T. Son and A.O. Starinets, Viscosity, black holes and Quantum Field Theory, Ann. Rev. Nucl. Part. Sci. 57 (2007) 95 [arXiv:0704.0240] [InSPIRE].

[3] E. Berti, V. Cardoso and A.O. Starinets, Quasinormal modes of black holes and black branes, Class. Quant. Grav. 26 (2009) 163001 [arXiv:0905.2975] [INSPIRE].

[4] L. Motl and A. Neitzke, Asymptotic black hole quasinormal frequencies, Adv. Theor. Math. Phys. 7 (2003) 307 [hep-th/0301173] [InSPIRE].

[5] A. Castro, J.M. Lapan, A. Maloney and M.J. Rodriguez, Black hole monodromy and Conformal Field Theory, Phys. Rev. D 88 (2013) 044003 [arXiv:1303.0759] [INSPIRE].

[6] A. Castro, J.M. Lapan, A. Maloney and M.J. Rodriguez, Black hole scattering from monodromy, Class. Quant. Grav. 30 (2013) 165005 [arXiv: 1304.3781] [INSPIRE].

[7] L.F. Alday, D. Gaiotto and Y. Tachikawa, Liouville correlation functions from four-dimensional gauge theories, Lett. Math. Phys. 91 (2010) 167 [arXiv:0906.3219] [INSPIRE].

[8] V.A. Alba, V.A. Fateev, A.V. Litvinov and G.M. Tarnopolskiy, On combinatorial expansion of the conformal blocks arising from AGT conjecture, Lett. Math. Phys. 98 (2011) 33 [arXiv: 1012.1312] [INSPIRE].

[9] L. Schlesinger, Über eine Klasse von Differentialsystemen beliebiger Ordnung mit festen kritischen Punkten (in German), J. Reine u. Angew. Math. 141 (1912) 96.

[10] K. Iwasaki, H. Kimura, S. Shimomura and M. Yoshida, From Gauss to Painlevé: a modern theory of special functions, Aspects Math. E 16, Braunschweig, Germany (1991).

[11] L.J. Mason, M.A. Singer and N.M.J. Woodhouse, Tau functions and the twistor theory of integrable systems, J. Geom. Phys. 32 (2000) 397.

[12] R.M. Wald, General relativity, The University of Chicago Press, Chicago U.S.A. (1984).

[13] V.P. Frolov, P. Krtous and D. Kubiznak, Separability of Hamilton-Jacobi and Klein-Gordon equations in general Kerr-NUT-AdS spacetimes, JHEP 02 (2007) 005 [hep-th/0611245] [INSPIRE].

[14] V.P. Frolov, Hidden symmetries and black holes, J. Phys. Conf. Ser. 189 (2009) 012015 [arXiv:0901.1472] [INSPIRE].

[15] J.B. Griffiths and J. Podolsky, On the parameters of the Kerr-NUT-(anti-)de Sitter space-time, Class. Quant. Grav. 24 (2007) 1687 [gr-qc/0702042] [INSPIRE].

[16] B. Carter et al., Hamilton-Jacobi and Schrödinger separable solutions of Einstein's equations, Commun. Math. Phys. 10 (1968) 280 [InSPIRE].

[17] C.M. Chambers and I.G. Moss, Stability of the Cauchy horizon in Kerr-de Sitter space-times, Class. Quant. Grav. 11 (1994) 1035 [gr-qc/9404015] [INSPIRE].

[18] M. Giammatteo and I.G. Moss, Gravitational quasinormal modes for Kerr anti-de Sitter black holes, Class. Quant. Grav. 22 (2005) 1803 [gr-qc/0502046] [INSPIRE].

[19] H. Suzuki, E. Takasugi and H. Umetsu, Perturbations of Kerr-de Sitter black hole and Heun's equations, Prog. Theor. Phys. 100 (1998) 491 [gr-qc/9805064] [INSPIRE]. 
[20] D. Batic and H. Schmid, Heun equation, Teukolsky equation and type-D metrics, J. Math. Phys. 48 (2007) 042502 [gr-qc/0701064] [InSPIRE].

[21] S.Y. Slavyanov and W. Lay, Special functions: a unified theory based on singularities, Oxford University Press, U.S.A. (2000).

[22] A.V. Shanin and R.V. Craster, Removing false singular points as a method of solving ordinary differential equations, Euro. J. Appl. Math. 13 (2002) 617.

[23] A. Ronveaux and F. Arscott, Heun's differential equations, Oxford University Press, (1995).

[24] Y.S. Choun, The analytic solution for the power series expansion of Heun function, Annals Phys. 338 (2013) 21 [arXiv:1303.0830].

[25] M. Jimbo, T. Miwa and A.K. Ueno, Monodromy preserving deformation of linear ordinary differential equations with rational coefficients, I, Physica D 2 (1981) 306.

[26] M. Jimbo and T. Miwa, Monodromy preserving deformation of linear ordinary differential equations with rational coefficients, II, Physica D 2 (1981) 407.

[27] M. Jimbo and T. Miwa, Monodromy preserving deformation of linear ordinary differential equations with rational coefficients, III, Physica D 4 (1981) 26.

[28] M. Jimbo, Monodromy problem and the boundary condition for some Painlevé equations, Publ. Res. Inst. Math. Sci. 18 (1982) 1137.

[29] K. Okamoto, Studies on Painlevé equations, Ann. Mat. Pura Appl. 146 (1986) 337.

[30] D. Guzzetti, A review on the sixth Painlevé equation, arXiv:1210.0311.

[31] N.J. Hitchin, Geometrical aspects of Schlesinger's equation, J. Geom. Phys. 23 (1997) 287 [INSPIRE].

[32] M.F. Atiyah and R. Bott, The Yang-Mills equations over Riemann surfaces, Phil. Trans. Roy. Soc. Lond. A 308 (1982) 523 [InSPIRE].

[33] A. Litvinov, S. Lukyanov, N. Nekrasov and A. Zamolodchikov, Classical conformal blocks and Painlevé VI, arXiv:1309.4700 [INSPIRE].

[34] I. Krichever, Isomonodromy equations on algebraic curves, canonical transformations and Whitham equations, hep-th/0112096 [INSPIRE].

[35] N. Nekrasov, A. Rosly and S. Shatashvili, Darboux coordinates, Yang-Yang functional and gauge theory, Nucl. Phys. Proc. Suppl. 216 (2011) 69 [arXiv:1103.3919] [InSPIRE].

[36] S.Y. Slavyanov, Painlevé equations as classical analogues of Heun equations, J. Phys. A 29 (1996) 7329.

[37] D.V. Anosov and A.A. Bolibruch, The Riemann-Hilbert problem, Aspects Math. E 22, Braunschweig, Germany (1994).

[38] V.G. Turaev, Skein quantization of Poisson algebras of loops on surfaces, Ann. Sci. Ecole Norm. Sup. (4) 24 (1991) 635704.

[39] A.B. Zamolodchikov, Conformal symmetry in two-dimensions: recursion representation of conformal block, Theor. Math. Phys. 53 (1987) 1088 [Teor. Mat. Fiz. 73 (1987) 103].

[40] A.B. Zamolodchikov and A.B. Zamolodchikov, Structure constants and conformal bootstrap in Liouville field theory, Nucl. Phys. B 477 (1996) 577 [hep-th/9506136] [INSPIRE]. 
[41] A. Mironov, A. Morozov and S. Shakirov, A direct proof of AGT conjecture at $\beta=1$, JHEP 02 (2011) 067 [arXiv: 1012.3137] [INSPIRE].

[42] O. Gamayun, N. Iorgov and O. Lisovyy, Conformal field theory of Painlevé VI, JHEP 10 (2012) 038 [Erratum ibid. 10 (2012) 183] [arXiv:1207.0787] [INSPIRE].

[43] N. Iorgov, O. Lisovyy and J. Teschner, Isomonodromic tau-functions from Liouville conformal blocks, arXiv:1401.6104 [INSPIRE].

[44] P.H. Ginsparg and G.W. Moore, Lectures on $2 D$ gravity and $2 D$ string theory, hep-th/9304011 [INSPIRE].

[45] V.I. Arnold, Mathematical methods of classical mechanics, Springer-Verlag, (1989).

[46] P. Boalch, From Klein to Painlevé via Fourier, Laplace and Jimbo, Proc. London Math. Soc. 90 (2005) 167 [math. AG/0308221].

[47] B.G. Carneiro da Cunha, Inflation and holography in string theory, Phys. Rev. D 65 (2002) 026001 [hep-th/0105219] [INSPIRE].

[48] J.M. Maldacena, Eternal black holes in anti-de Sitter, JHEP 04 (2003) 021 [hep-th/0106112] [INSPIRE].

[49] L. Fidkowski, V. Hubeny, M. Kleban and S. Shenker, The black hole singularity in AdS/CFT, JHEP 02 (2004) 014 [hep-th/0306170] [INSPIRE].

[50] L. Bers and I. Kra eds., A crash course on Kleinian groups, Lect. Notes Math. 400, Springer-Verlag, (1974).

[51] A.R. Forsyth, Theory of differential equations, III, Cambridge University Press, (1902).

[52] D. Guzzetti, Tabulation of Painlevé 6 transcendents, Nonlinearity 25 (2012) 3235 [arXiv: 1108.3401].

[53] P. Menotti, On the monodromy problem for the four-punctured sphere, arXiv:1401.2409 [INSPIRE]. 